\title{
Edge Disjoint Hamilton Cycles in Knödel Graphs
}

\author{
P. Paulraja ${ }^{1 *}$ \\ S. Sampath $\operatorname{Kumar}^{2 \dagger}$ \\ 1 Department of Mathematics, Kalasalingam University, Krishnankoil, India \\ 2 Department of Mathematics, SSN College of Engineering, Kalavakkam, India
}

received $31^{\text {st }}$ July 2014, revised $3^{\text {rd }}$ Aug. 2015, 24 th June 2016, accepted $7^{\text {th }}$ July 2016.

The vertices of the Knödel graph $W_{\Delta, n}$ on $n \geq 2$ vertices, $n$ even, and of maximum degree $\Delta, 1 \leq \Delta \leq\left\lfloor\log _{2}(n)\right\rfloor$, are the pairs $(i, j)$ with $i=1,2$ and $0 \leq j \leq \frac{n}{2}-1$. For $0 \leq j \leq \frac{n}{2}-1$, there is an edge between vertex $(1, j)$ and every vertex $\left(2, j+2^{k}-1\left(\bmod \frac{n}{2}\right)\right)$, for $k=0,1,2, \ldots, \Delta-1$. Existence of a Hamilton cycle decomposition of $W_{k, 2^{k}}, k \geq 6$ is not yet known, see Discrete Appl. Math. 137 (2004) 173-195. In this paper, it is shown that the $k$-regular Knödel graph $W_{k, 2^{k}}, k \geq 6$ has $\left\lfloor\frac{k}{2}\right\rfloor-1$ edge disjoint Hamilton cycles.

Keywords: Knödel Graphs, Hamilton Cycle Decomposition, Tensor Product, Bieulerian Graph

\section{Introduction.}

All graphs considered here are simple and finite unless otherwise stated. Let $C_{k}$ (resp. $P_{k}$ ) denote the cycle (resp. path) on $k$ vertices. For a graph $G$, if its edge set $E(G)$ can be partitioned into $E_{1}, E_{2}, \ldots, E_{k}$ such that $\left\langle E_{i}\right\rangle \cong H$, for all $i, 1 \leq i \leq k$, then we say that $H$ decomposes $G$. A $k$-factor of $G$ is a $k$-regular spanning subgraph of it. A k-factorization of a graph $G$ is a partition of the edge set of $G$ into $E_{1}, E_{2}, \ldots, E_{s}$ such that $\left\langle E_{i}\right\rangle, 1 \leq i \leq s$, is a $k$-factor. We say that a $k$-regular graph $G$ admits a Hamilton cycle decomposition, if the edge set of $G$ can be partitioned into Hamilton cycles or Hamilton cycles together with a 1-factor according as $k$ is even or odd, respectively. If $H_{1}, H_{2}, \ldots, H_{k}$ are edge disjoint subgraphs of $G$ such that $\bigcup_{i=1}^{k} H_{i}=G$, then we write $G=H_{1} \oplus H_{2} \oplus \ldots \oplus H_{k}$. The complete graph on $n$ vertices is denoted by $K_{n}$. Let $G$ be a bipartite graph with bipartition $(X, Y)$, where $X=\left\{x_{0}, x_{1}, \ldots, x_{n-1}\right\}$, $Y=\left\{y_{0}, y_{1}, \ldots, y_{n-1}\right\}$; the edge $x_{i} y_{i+\ell}$ is called an edge of jump $\ell$ from $X$ to $Y$ in $G$, where addition is taken modulo $n$; the same edge is called an edge of jump $n-\ell$ from $Y$ to $X$. If $G$ contains the edges $F_{\ell}(X, Y)=\left\{x_{i} y_{i+\ell} \mid 0 \leq i \leq n-1\right.$, where addition in the subscript is taken modulo $\left.n\right\}, 0 \leq$ $\ell \leq n-1$, then we say that $G$ has the 1-factor of jump $\ell$ from $X$ to $Y$. Clearly, if $G=K_{n, n}$, then $E(G)=\bigcup_{i=0}^{n-1} F_{i}(X, Y)$. Note that $F_{i}(X, Y)=F_{n-i}(Y, X), 0 \leq i \leq n-1$, where we assume $F_{n}(X, Y)=F_{0}(X, Y)=F_{0}(Y, X)$.

An anti-directed path $P$ is a digraph, whose underlying graph is a path, in which any two consecutive arcs of $P$ are either directed toward or away from the common incident vertex in $P$. Similarly, we define anti-directed cycles, see Figures $1($ a) and $1(\mathrm{~b})$. A digraph $\vec{G}=(V, A)$ is denoted by $\vec{G}$. A digraph

\footnotetext{
*ppraja56@gmail.com

†sampathkumars@ssn.edu.in

1365-8050 @ 2016 Discrete Mathematics and Theoretical Computer Science (DMTCS), Nancy, France
} 
$\vec{G}=(V, A)$ is said to be $k$-diregular if $d^{+}(x)=k=d^{-}(x)$ for every $x \in V$. If $x$ is the tail, and $y$ is the head of an arc of $\vec{G}$, then it is denoted by $\overrightarrow{x y}$. A digraph $\vec{G}$ is called aneulerian if $\vec{G}$ has an Euler tour $T$ such that any two consecutive edges of $T$ are either directed toward or away from the common incident vertex in $\vec{G}$, see Figures 1(c) and 1(d). A directed graph is called bieulerian if it is both eulerian (that is, it contains a directed Euler tour) and aneulerian, see Figures 1(c) and 1(d); consequently, a digraph which admits an aneulerian tour cannot be $k$-diregular for $k \geq 3$.

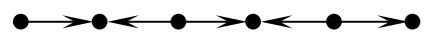

(a). An anti-directed path

on 6 vertices

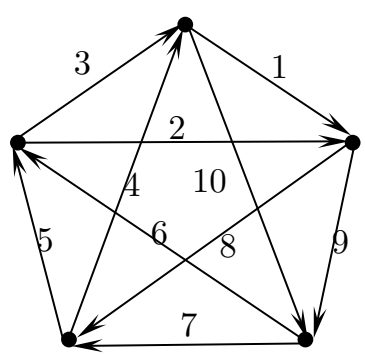

(c). An aneulerian tour in $\vec{K}_{5}$ is described by the arc sequence

$1,2,3, \ldots, 10$, taken in that order.

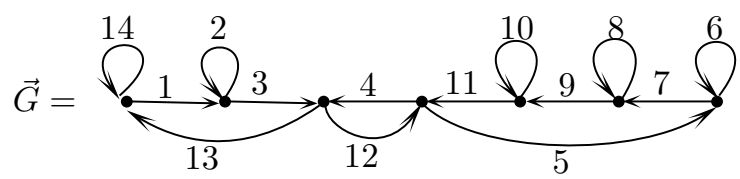

(d) An aneulerian tour in $\vec{G}$ with directed loops at some of its vertices is given by the arc sequence $1,2, \ldots, 13,14$, where the directed loops are traversed in the forward or backward direction in order to have an aneulerian tour in $\vec{G}$.

Fig. 1: Examples for anti-directed path, anti-directed cycle and aneulerian tours.

For two graphs $G$ and $H$ their tensor product, denoted by $G \times H$, has vertex set $V(G) \times V(H)$ in which $\left(g_{1}, h_{1}\right)\left(g_{2}, h_{2}\right)$ is an edge in $G \times H$ whenever $g_{1} g_{2} \in E(G)$ and $h_{1} h_{2} \in E(H)$. Similarly, for two digraphs $\vec{G}$ and $\vec{H}$ their tensor product, denoted by $\vec{G} \times \vec{H}$, has vertex set $V(\vec{G}) \times V(\vec{H})$ in which 
$\overrightarrow{\left(g_{1}, h_{1}\right)\left(g_{2}, h_{2}\right)}$ is an arc in $\vec{G} \times \vec{H}$ whenever $\overrightarrow{g_{1} g_{2}} \in A(\vec{G})$ and $\overrightarrow{h_{1} h_{2}} \in A(\vec{H})$, where $A(\vec{G})$ denotes the arc set of $\vec{G}$. Note that if $x x$ is a loop at $x$ in $G$ (resp. $H$ ), then $x \times V(H)$ (resp. $V(G) \times x)$ induces a copy of $H$ (resp. $G$ ) in $G \times H$. A circulant graph $X=\operatorname{Circ}(n ; L)$ is a graph with vertex set $V(X)=$ $\left\{u_{0}, u_{1}, \ldots, u_{n-1}\right\}$ and edge set $E(X)=\left\{u_{i} u_{i+\ell} \mid i \in \mathbb{Z}_{n}, \ell \in L\right\}$, where $L \subseteq\left\{1,2, \ldots,\left\lfloor\frac{n}{2}\right\rfloor\right\}$. The elements of $L$ are called distances of the circulant graph and $L$ is called the set of distances. A circulant digraph $\vec{X}=\overrightarrow{\operatorname{Circ}}(n ; L)$ is a digraph with vertex set $V(\vec{X})=\left\{u_{0}, u_{1}, \ldots, u_{n-1}\right\}$ and arc set $A(\vec{X})=\left\{\overrightarrow{u_{i} u_{i+\ell}} \mid i \in \mathbb{Z}_{n}, \ell \in L\right\}$, where $L \subseteq\{1,2, \ldots, n-1\}$. The elements of $L$ are called distances of the circulant digraph. The underlying graph of a digraph $\vec{D}$ is the undirected graph obtained from $\vec{D}$ by simply deleting the orientations of the arcs of $\vec{D}$. For graph theoretical terms not defined here, see $[2,6]$.

Knödel graph was originally introduced in [22]. The family of Knödel graphs has been formally defined by Fraigniaud and Peters [7]. Knödel graph $W_{\Delta, n}$ is a regular graph of even order $n$ and degree $\Delta, 1 \leq \Delta \leq\left\lfloor\log _{2}(n)\right\rfloor$. Knödel graphs are used as competitors for hypercubes in the domains of broadcasting and gossiping. The gossiping problem, as described by Knödel in [22] is the following: "Given $n$ persons, each having an information, want to distribute their information among them in binary calls, each call taking a constant time, how long must it take before each knows all the information among them?" Broadcasting is a similar problem where only one person (the originator) has all the information that needs to be distributed to a group of people in binary calls. Consequently, they deal with problems in dissemination of information in interconnection networks. Every interconnection network can be represented by means of a graph. If this graph has $n$ vertices, the minimum time required for broadcasting is $\left\lceil\log _{2} n\right\rceil$. Such graphs are known as minimal broadcasting graphs. For more details on minimal broadcasting and gossiping graphs, see $[11,19,20]$. There are several papers dealing with Knödel graphs, especially because some subfamilies of Knödel graphs have good properties in terms of broadcasting and gossiping and Fault-Tolerance, see $[1,10,14,18,21,23]$. In particular, for $n=2^{k}$, the Knödel graph $W_{k, 2^{k}}$, of order $n$ and degree $k$, turns out to be a minimum broadcast graph. It is known that, the diameter of $W_{k, 2^{k}}$ is $\left\lceil\frac{k+2}{2}\right\rceil$, see [9]. It is known that $W_{k, 2^{k}}$ is vertex-transitive but not edge-transitive, see [8]. Recently, it has been proved, see [4], that the automorphism group of $W_{k, 2^{k}}$ is the dihedral group $D_{2^{k-1}}$ and in the same paper a short proof is given for the diameter of the Knödel graph $W_{k, 2^{k}}$. For other properties of the Knödel graphs and modified Knödel graphs, see [5, 12,13,15-17].

It is known that $W_{k-1,2^{k}-2}$ is Hamilton cycle decomposable and $W_{k, 2^{k}}$ is bipancyclic, that is, every cycle of length $\ell, 4 \leq \ell \leq 2^{k}$ exists, see [8]. A detailed account of various properties the Knödel graphs and comparison with other interconnection networks has been given in the survey [8].

It is not yet known whether the Knödel graphs $W_{k, 2^{k}}, k \geq 6$ admit a Hamilton cycle decomposition or not [8]. In this paper, we prove that the $k$-regular Knödel graph $W_{k, 2^{k}}, k \geq 6$ has $\left\lfloor\frac{k}{2}\right\rfloor-1$ edge disjoint Hamilton cycles, that is, the edge set of $W_{k, 2^{k}}, k \geq 6$ can be partitioned into $\left\lfloor\frac{k}{2}\right\rfloor-1$ Hamilton cycles together with a 2 -factor, if $k$ is even and $\left\lfloor\frac{k}{2}\right\rfloor-1$ Hamilton cycles together with a 3 -factor, if $k$ is odd.

\section{Definitions and Preliminaries.}

Definition 2.1 [8] The Knödel graph on $n \geq 2$ vertices, $n$ even, and maximum degree $\Delta, 1 \leq \Delta \leq$ $\left\lfloor\log _{2}(n)\right\rfloor$, is denoted by $W_{\Delta, n}$. The vertices of $W_{\Delta, n}$ are the pairs $(i, j)$ with $i=1,2$ and $0 \leq j \leq$ $\frac{n}{2}-1$. For every $0 \leq j \leq \frac{n}{2}-1$, there is an edge between vertex $(1, j)$ and every vertex $(2, j+$ $\left.2^{k}-1\left(\bmod \frac{n}{2}\right)\right)$, for $k=0,1,2, \ldots, \Delta-1$. It is a bipartite graph containing the jump 1-factors 
$2^{k}-1, k=0,1,2, \ldots, \Delta-1$.

For each $k, 0 \leq k \leq \Delta-1$, the edges $(1, j)\left(2, j+2^{k}-1\left(\bmod \frac{n}{2}\right)\right), 0 \leq j \leq \frac{n}{2}-1$, induce a 1 -factor of jump $2^{k}-1$ in $W_{\Delta, n}$.

The union of the 1 -factors of jumps 0 and 1 from $X$ to $Y$ of $W_{\Delta, n}$ is a Hamilton cycle, where we assume that $X=\left\{(1, j) \mid 0 \leq j \leq \frac{n}{2}-1\right\}$ and $Y=\left\{(2, j) \mid 0 \leq j \leq \frac{n}{2}-1\right\}$ are the bipartition of $W_{\Delta, n}$. It is known that the graphs $W_{k, 2^{k}}, k \leq 5$, are Hamilton cycle decomposable, see [8]. Based on this observation the following problem is raised in [8].

Problem 2.1 [8] Is $W_{k, 2^{k}}$ Hamilton cycle decomposable for any $k \geq 2$ ?

In this paper, we prove that the $k$-regular Knödel graph $W_{k, 2^{k}}, k \geq 6$ has $\left\lfloor\frac{k}{2}\right\rfloor-1$ edge disjoint Hamilton cycles.

Definition 2.2 (Bipartite incident graph [8]). Let $\vec{G}=(V, A)$ be a digraph of order $n$, with $V=$ $\{0,1, \ldots, n-1\}$. The bipartite incident graph of $\vec{G}$ is the (undirected) bipartite graph $H=\left(V_{1}, V_{2}, E\right)$ of order $2 n$, where $V_{i}=\left\{0_{i}, 1_{i}, \ldots,(n-1)_{i}\right\}, i=1,2$, and that for any arc $\overrightarrow{i j}$ of $\vec{G}$, there corresponds an edge $i_{1} j_{2} \in E(H)$ with $i_{1} \in V_{1}$ and $j_{2} \in V_{2}$ and for each $i \in V$, there is an edge $i_{1} i_{2} \in E(H)$, where $i_{1} \in V_{1}$ and $i_{2} \in V_{2}$ in $H$.

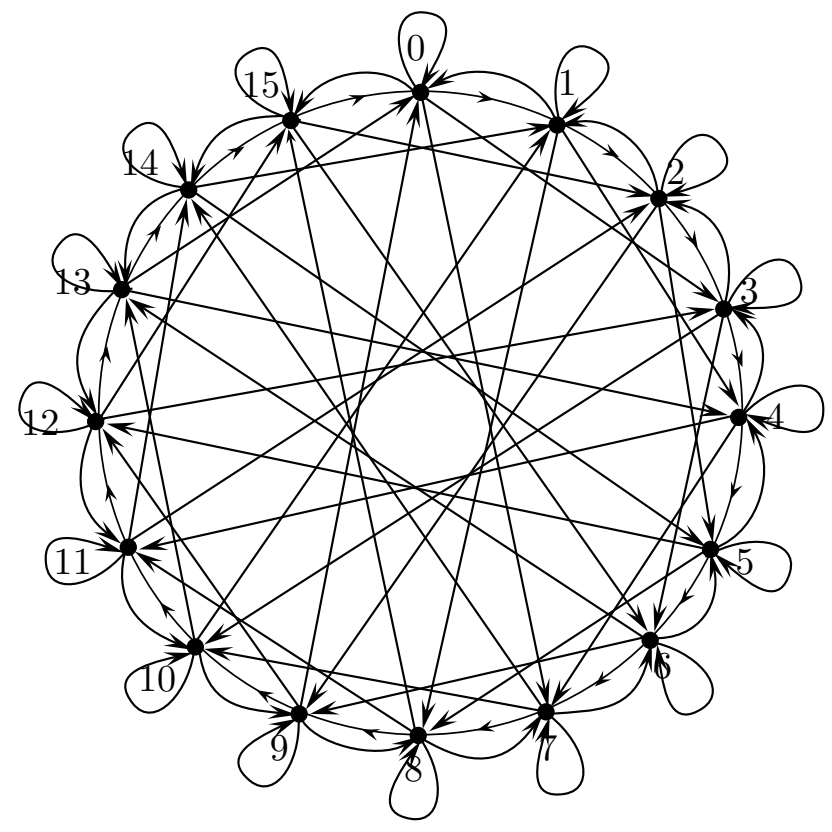

Fig. 2: The Circulant digraph $\vec{G}_{5}^{\prime}=\overrightarrow{\operatorname{Circ}}(16,\{0,1,3,7,15\})$. 
From the Definition 2.2, it is easy to see that the Knödel graph $W_{\Delta, n}$ is the bipartite incident graph of the circulant digraph $\vec{G}$ with vertex set $\left\{0,1,2, \ldots, \frac{n}{2}-1\right\}$ and arc set consists of the arcs of distance $\ell$, $\ell \in L=\left\{2^{k}-1,0 \leq k \leq \Delta-1\right\}$. In otherwords, $W_{\Delta, n}$ is the underlying graph of $\vec{G} \times \vec{K}_{2}$, where $\vec{K}_{2}$ denotes an arc.

Let $\vec{G}_{k}$ denote the circulant digraph $\overrightarrow{\operatorname{Circ}}\left(2^{k-1} ; L\right)$, where $L=\left\{2^{j}-1 \mid 1 \leq j \leq k-1\right\}$ and let $\vec{G}_{k}^{\prime}$ denote the graph obtained from $\vec{G}_{k}$ by attaching a directed loop at each of its vertices, that is, $\vec{G}_{k}^{\prime}=\overrightarrow{\operatorname{Circ}}\left(2^{k-1} ; L \cup\{0\}\right)$, see Figure 2. Clearly, $W_{k, 2^{k}}$ is isomorphic to the underlying graph of $\vec{G}_{k}^{\prime} \times \vec{K}_{2}$

Definition 2.3 [3] Let $\vec{G}$ be a 2-diregular digraph which does not have a pair of arcs having the same tail and head, but may have directed loops. If $\vec{G}$ admits an uniformly odd aneulerian tour $(U O A T)$, that is, an aneulerian tour in which no proper closed subtrail is of even length, then $\vec{G}$ is called an UOAT digraph (in fact, one can check that if a 2-diregular digraph admits an aneulerian tour $T$, then each proper closed subtrail of $T$ is of odd length).

The proof of the following theorem is similar to the proof of Theorem 2.1 of [3].

Theorem 2.1 Let $\vec{G}$ be a 2-diregular digraph which does not have a pair of arcs having the same tail and head, but may have directed loops. Then the underlying graph of the digraph $\vec{G} \times \vec{K}_{2}$ is a Hamilton cycle if and only if $\vec{G}$ is an UOAT digraph.

Proof: Let $\vec{G}=(V, A)$ with $V(\vec{G})=\left\{v_{0}, v_{1}, v_{2}, \ldots, v_{n}\right\}$. Let $\vec{K}_{2}=\overrightarrow{x y}$. To each arc $\overrightarrow{v_{i} v_{j}}$, possibly with $i=j$, in $\vec{G}$ there corresponds an unique arc $\overrightarrow{\left(v_{i}, x\right)\left(v_{j}, y\right)}$ in $\vec{G} \times \vec{K}_{2}$ and similarly corresponding to each arc $\overrightarrow{\left(v_{r}, x\right)\left(v_{s}, y\right)}$, possibly with $r=s$, in $\vec{G} \times \vec{K}_{2}$ there corresponds an arc $\overrightarrow{v_{r} v_{s}}$, may be a directed loop, in $\vec{G}$. Clearly, $\vec{H}=\vec{G} \times \vec{K}_{2}$ is bipartite digraph with bipartition $(X, Y)$, where $X=V(\vec{G}) \times x=$ $\left\{\left(v_{i}, x\right) \mid v_{i} \in V(\vec{G})\right\}$ and $Y=V(\vec{G}) \times y=\left\{\left(v_{i}, y\right) \mid v_{i} \in V(\vec{G})\right\}$. In $\vec{H}$ all the vertices in $X$ have only outdegree, namely 2 , and all the vertices in $Y$ have only indegree 2 . Hence the underlying graph of $\vec{G} \times \vec{K}_{2}$ is a 2-regular graph. First assume that the underlying graph of $\vec{G} \times \vec{K}_{2}$ is a spanning cycle $C$. As each vertex in $X$ has only outdegree and each vertex in $Y$ has only indegree, while we trace along $C$ the corresponding arcs in $\vec{G}$ trace an anti-directed tour $T$. We claim that $T$ is an aneulerian tour. Observe that if $\left(v_{i}, x\right)\left(v_{i}, y\right)$ is an edge of $C$, then its corresponding arc in $T$ is a directed loop at $v_{i}$, see Figure 3 . We claim that no proper closed subtrail of $T$ is of even length. Let $v_{k}$ be the origin of a proper closed subtrail $T_{1}$ of $T$. Without loss of generality assume that $C$ visits $\left(v_{k}, x\right)$ prior to $\left(v_{k}, y\right)$. Hence $T_{1}$ arises out of the $\left(v_{k}, x\right)$ - $\left(v_{k}, y\right)$ section of $C$ and this section is of odd length as $\vec{G} \times \vec{K}_{2}$ is a bipartite digraph. Hence $T_{1}$ is of odd length. Hence $T$ is an aneulerian tour. Thus $\vec{G}$ is an $U O A T$ digraph.

Conversely, assume that $\vec{G}$ is an $U O A T$ digraph. Let $T$ be an aneulerian tour of $\vec{G}$ with the origin $v_{0}$. Now we describe a Hamilton cycle in the underlying graph of $\vec{G} \times \vec{K}_{2}$ from the vertex $\left(v_{0}, x\right)$ as follows: If $\overrightarrow{v_{0} v_{1}}$ is the first arc of $T$, then take the edge $\left(v_{0}, x\right)\left(v_{1}, y\right)$ in the underlying graph of $\vec{G} \times \vec{K}_{2}$ for the Hamilton cycle. The second arc of $T$ would be $\overrightarrow{v_{2} v_{1}}$, then take the edge $\left(v_{1}, y\right)\left(v_{2}, x\right)$ in the underlying graph of $\vec{G} \times \vec{K}_{2}$. As we move along $T$, the corresponding edges of the underlying graph of $\vec{G} \times \vec{K}_{2}$ induce a Hamilton cycle in the underlying graph of $\vec{G} \times \vec{K}_{2}$; for otherwise, the corresponding arcs of an nonspanning even cycle in the underlying graph of $\vec{G} \times \vec{K}_{2}$ would yield a proper closed subtrail of even length in $T$, a contradiction. Hence as we trace along $T$, the corresponding edges of the underlying graph of $\vec{G} \times \vec{K}_{2}$ induce a Hamilton cycle. 


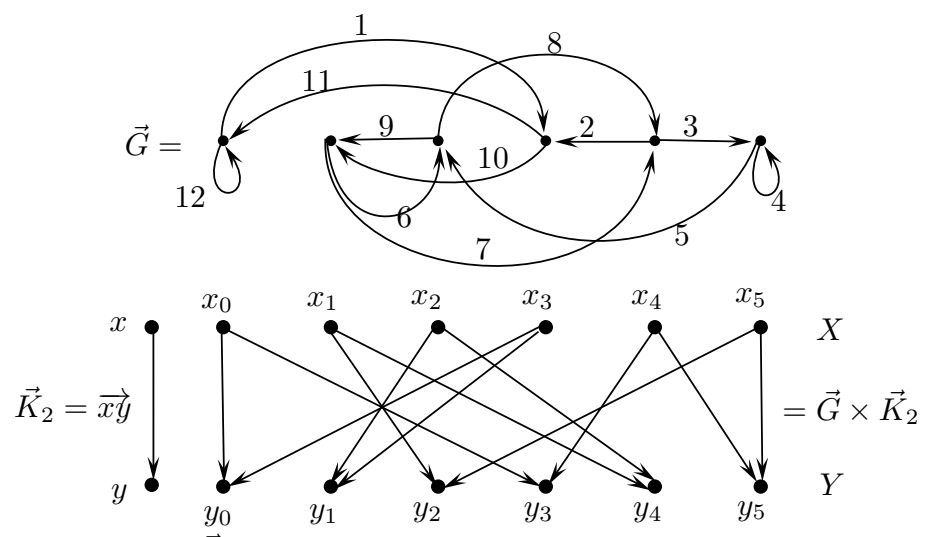

(i) A Directed Euler tour in $\vec{G}$ is given by the arc sequence $1,10,7,3,4,5,9,6,8,2,11,12$.

(ii) An aneulerian tour in $\vec{G}$ is given by the arc sequence $1,2,3, \ldots, 11,12$, where the loops are traversed in the forward or backward direction according as our requirement for the existence of an aneulerian tour. Clearly this aneulerian tour is an $U O A T$ of $\vec{G}$.

Fig. 3: $\vec{G} \times \vec{K}_{2}$, a 2-diregular bieulerian digraph.

This completes the proof of the theorem.

Theorem 2.2 Let $\vec{G}$ be a 2-diregular digraph which does not have a pair of arcs having the same tail and head, but may have directed loops. Then the underlying graph of $\vec{G} \times \vec{K}_{2}$ is a Hamilton cycle if and only if $\vec{G}$ is bieulerian.

Proof: First assume that the underlying graph $\vec{G} \times \vec{K}_{2}$ is a Hamilton cycle $C$. Then by Theorem $2.1 \vec{G}$ has an $U O A T$. As $\vec{G}$ is diregular, $\vec{G}$ is eulerian. Thus $\vec{G}$ is bieulerian.

Conversely, assume that $\vec{G}$ is bieulerian. Let $T$ be an aneulerian tour of $\vec{G}$. If we proceed as in the proof of Theorem 2.1, when we move along the aneulerian tour $T$, the corresponding edges in the underlying graph of $\vec{G} \times \vec{K}_{2}$ induce a Hamilton cycle.

This completes the proof of the theorem.

\section{Edge Disjoint Hamilton Cycles of $W_{k, 2^{k}}$.}

In this section we prove that $W_{k, 2^{k}}$ contains $\left\lfloor\frac{k}{2}\right\rfloor-1$ edge disjoint Hamilton cycles.

The following Theorem 3.1 is proved in [8].

Theorem 3.1 [8] For any even $m$ and $1 \leq \Delta \leq\left\lfloor\log _{2}(m)\right\rfloor$, it is possible to construct $W_{\Delta+1,2 m}$ by taking two disjoint copies of $W_{\Delta, m}$ and linking the vertices of the copies by a certain perfect matching. $\square$

Next we decompose the digraph $\vec{G}_{k}=\overrightarrow{\operatorname{Circ}}\left(2^{k-1}, L\right)$, where $L=\left\{2^{j}-1 \mid 1 \leq j \leq k-1\right\}$, into two digraphs in the following way to proceed further. Let $V\left(\vec{G}_{k}\right)=\left\{0,1,2, \ldots, 2^{k-1}-1\right\}$. First we decompose $\vec{G}_{k}$ into two digraphs $\vec{G}_{k, e}$ and $\vec{G}_{k, o}$, where $\vec{G}_{k, e}$ (resp. $\vec{G}_{k, o}$ ) is the spanning subdigraph 


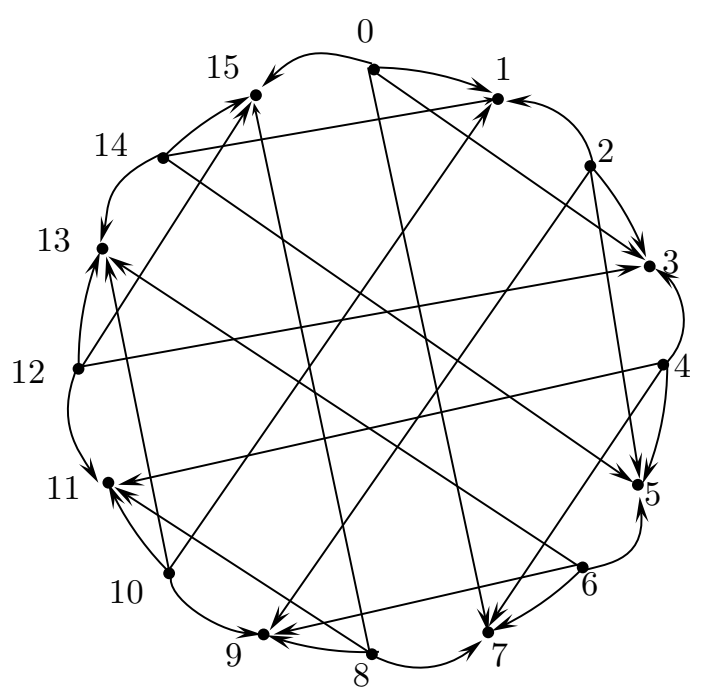

(a). $\vec{G}_{5, e}$

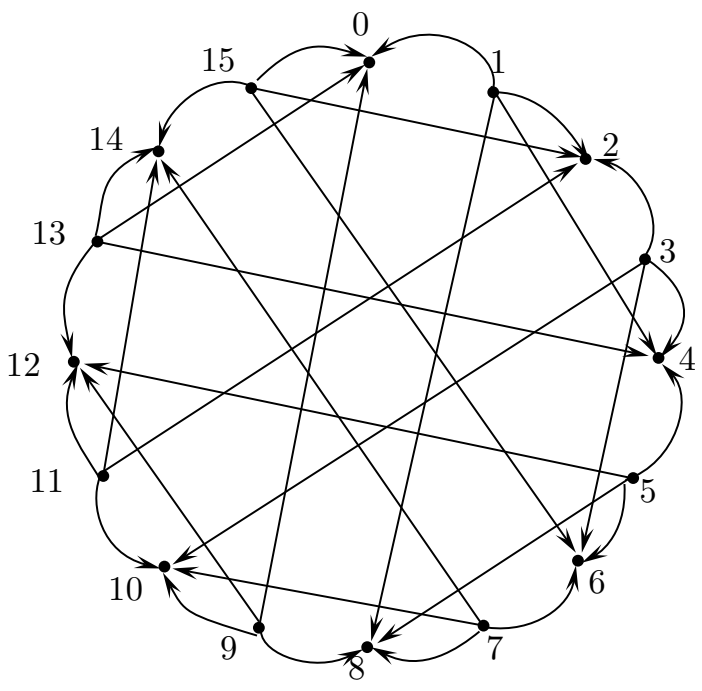

(b). $\vec{G}_{5, o}$

Fig. 4: Graphs $\vec{G}_{5, e}$ and $\vec{G}_{5, o}$.

of $\vec{G}_{k}$ in which the indegree of each of the even (resp. odd) vertices, namely, $0,2, \ldots, 2^{k-1}-2$ (resp. $1,3, \ldots, 2^{k-1}-1$ ), is zero. That is $\vec{G}_{k, e}$ (resp. $\vec{G}_{k, o}$ ) is the spanning subdigraph of $\vec{G}_{k}$ obtained by deleting the set of arcs having their tails at the odd (resp. even) vertices of $\vec{G}_{k}$. Each arc of $\vec{G}_{k, e}$ is directed from an even vertex to an odd vertex and each $\operatorname{arc}$ of $\vec{G}_{k, o}$ is directed from an odd vertex to an even vertex (see Figure 4 for $k=5$ ).

Lemma 3.1 The underlying graphs of the digraphs $\vec{G}_{k, e}$ and $\vec{G}_{k, o}$ are isomorphic to $W_{k-1,2^{k-1}}$.

Proof: $\vec{G}_{k, e}$ can be viewed as a bipartite graph by placing the even vertices, in the increasing order, in the partite set $X$ and the odd vertices, in the increasing order, in the other partite set $Y$. Then the arcs of distance $2^{\ell}-1,1 \leq \ell \leq k-1$, in $\vec{G}_{k, e}$, considering it as a subdigraph of $\vec{G}_{k}$, become the arcs of jump $2^{\ell-1}-1$ from $X$ to $Y$ in $\vec{G}_{k, e}$ in the bipartite structure of it and thus the underlying graph of $\vec{G}_{k, e}$ is isomorphic to $W_{k-1,2^{k-1}}$, see Figure 5(a), when $k=5$. To prove the underlying graph of $\vec{G}_{k, o}$ is isomorphic to $W_{k-1,2^{k-1}}$, we establish an isomorphism between $\vec{G}_{k, e}$ and $\vec{G}_{k, o}$ by mapping the vertex $i$ of $\vec{G}_{k, e}$ to $i+1$, where the addition is taken modulo $2^{k-1}$, of $\vec{G}_{k, o}$.

Recall that $\vec{G}_{k}^{\prime}$ is the digraph obtained from $\vec{G}_{k}$ by adding a loop at each of its vertices and, $W_{k, 2^{k}}$ is isomorphic to the underlying graph of $\vec{G}_{k}^{\prime} \times \vec{K}_{2}$.

First we explain the proof technique of Theorem 3.2 and the lemmas used in its proof. By Theorem 2.2, finding $\left\lfloor\frac{k}{2}\right\rfloor-1$ Hamilton cycles of Knödel graph $W_{k, 2^{k}}$ is equivalent to finding $\left\lfloor\frac{k}{2}\right\rfloor-1$ arc-disjoint spanning 2-diregular subdigraphs of $\vec{G}_{k}^{\prime}$ so that each of the subdigraphs is bieulerian. Hence we shall 


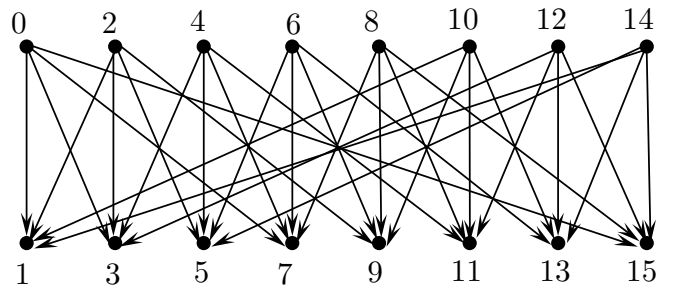

(a)

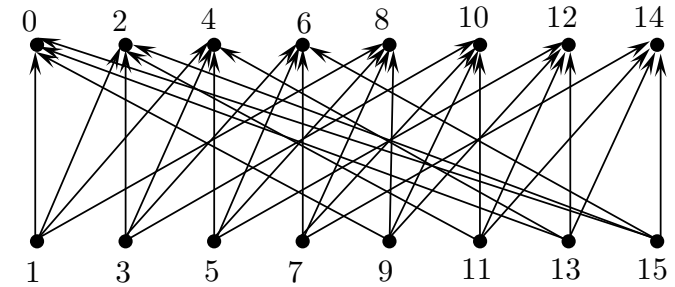

(b)

Fig. 5: Redrawings of $\vec{G}_{5, e}$ and $\vec{G}_{5, o}$, in Figure 4, in the "natural" bipartite structure. For example the edges of distance 7 in $\vec{G}_{5}, e$ are edges of jump 3 from $X$ to $Y$, where $X=\{0,2,4, \ldots, 14\}$ and $Y=\{1,3,5, \ldots, 15\}$, in the bipartite structure. Actually, an edge of distance $2^{\ell}-1$ in $\vec{G}_{k, e}$ becomes an edge of jump $2^{\ell-1}-1$, from $X$ to $Y$, in the bipartite structure. As the graphs of Figures 4(a) and 4(b) are isomorphic, the graphs in Figures 5(a) and 5(b) are isomorphic (the isomorphism is obtained by mapping the vertex $i$ in Figure 5 (a) to $i+1\left(\bmod 2^{k-1}\right)$ of Figure $5(\mathrm{~b}))$.

obtain $\left\lfloor\frac{k}{2}\right\rfloor-1$ arc-disjoint spanning 2-diregular subdigraphs of $\vec{G}_{k}^{\prime}$ such that each of which is bieulerian, if $k$ is even, and with a directed 1-factor (that is, $d^{+}(v)=d^{-}(v)=1$ for each $v$ ) if $k$ is odd. To establish this, in Lemma 3.2, first we shall obtain $\left\lfloor\frac{k}{2}\right\rfloor-1$ subdigraphs $\vec{H}_{i, e}, 1 \leq i \leq\left\lfloor\frac{k}{2}\right\rfloor-1$, and another $\left\lfloor\frac{k}{2}\right\rfloor-1$, subdigraphs $\vec{H}_{i, o}, 1 \leq i \leq\left\lfloor\frac{k}{2}\right\rfloor-1$ out of $\vec{G}_{k, e}$ and $\vec{G}_{k, o}$, respectively. Obtain the digraph $\vec{H}_{i}=\vec{H}_{i, e} \cup \vec{H}_{i, o}, 1 \leq i \leq\left\lfloor\frac{k}{2}\right\rfloor-1$. We shall prove that $\vec{H}_{i}, 1 \leq i \leq\left\lfloor\frac{k}{2}\right\rfloor-1$, together with some "appropriate" directed loops at some of its vertices is a spanning 2-diregular bieulerian subdigraph of $\vec{G}_{k}^{\prime}$, see Lemma 3.3. Using this, in Theorem 3.2, we prove the existence of $\left\lfloor\frac{k}{2}\right\rfloor-1$ edge disjoint Hamilton cycles in $W_{k, 2^{k}}$.

We shall prove what we have told above in the rest of the paper.

Remark 3.1 Let $G$ be a bipartite graph with bipartition $(X, Y)$ and $|X|=|Y|=n$. Let $F_{i}(X, Y)$ and $F_{j}(X, Y), i \neq j$, be two 1-factors of jump $i$ and $j$, respectively, from $X$ to $Y$ in $G$. Let $H=$ $F_{i}(X, Y) \cup F_{j}(X, Y)$. Note that $F_{j}(X, Y)=F_{n-j}(Y, X)$. If $\operatorname{gcd}(n, i+n-j(\bmod n))=k$, then $H$ is the union of $k$ disjoint cycles of same length. We shall use this fact in the proof of the next lemma; in particular, if $\operatorname{gcd}(n, i+n-j(\bmod n))=1$, then $F_{i}(X, Y) \cup F_{j}(X, Y)$ is a Hamilton cycle of $G$.

Lemma 3.2 There exist $\left\lfloor\frac{k}{2}\right\rfloor-1$ arc-disjoint spanning subdigraphs $\vec{H}_{j, e}, 1 \leq j \leq\left\lfloor\frac{k}{2}\right\rfloor-1$, of $\vec{G}_{k, e}$, where each $\vec{H}_{j, e}$ is the union of $2^{j-1}$ disjoint anti-directed paths whose origins and termini be denoted by $A_{j}\left(\subset V\left(\vec{G}_{k}\right)\right)$, and, also there exists $\left\lfloor\frac{k}{2}\right\rfloor-1$ arc-disjoint spanning subdigraphs $\vec{H}_{j, o}, 1 \leq j \leq\left\lfloor\frac{k}{2}\right\rfloor-1$, of $\vec{G}_{k, o}$, where each $\vec{H}_{j, o}$ is the union of $2^{j-1}$ disjoint anti-directed paths whose origins and termini are also $A_{j}$; that is, the set of origins and termini of the anti-directed paths of $\vec{H}_{j, e}$ is the same as the set of origins and termini of the anti-directed paths of $\vec{H}_{j, o}$; moreover $A_{i} \cap A_{j}=\phi$ for $i \neq j$.

Proof: $\vec{G}_{k}$ is the circulant digraph with distance set $\left\{2^{j}-1 \mid 1 \leq j \leq k-1\right\}$. Let $\vec{G}_{k, e}$ and $\vec{G}_{k, o}$ be the spanning subdigraphs of $\vec{G}_{k}$ defined as above.

Partition the distance set $\left\{2^{j}-1 \mid 1 \leq j \leq k-2\right\}$ of $\vec{G}_{k}$ into $\left\lfloor\frac{k}{2}\right\rfloor-12$-subsets $\left\{a_{j}=2^{j}-1, b_{j}=\right.$ $\left.2^{k-1-j}-1\right\}, 1 \leq j \leq\left\lfloor\frac{k}{2}\right\rfloor-1$, if $k$ is even and, with one more singleton subset having only one distance, 
namely $2^{\frac{k-1}{2}}-1$, if $k$ is odd. For each of these pairs of distances, we shall obtain a pair of spanning subdigraphs $\vec{H}_{j, e}$ and $\vec{H}_{j, o}$ of $\vec{G}_{k, e}$ and $\vec{G}_{k, o}$, respectively, such that each of the digraphs $\vec{H}_{j, e}$ and $\vec{H}_{j, o}$ is the union of $2^{j-1}$ vertex disjoint anti-directed paths.

Let $\vec{G}_{k, e}^{j}, 1 \leq j \leq\left\lfloor\frac{k}{2}\right\rfloor-1$, be the spanning subdigraph of $\vec{G}_{k, e}$, with arc set $A\left(\vec{G}_{k, e}\right) \cap A\left(\overrightarrow{\operatorname{Circ}}\left(2^{k-1} ;\left\{2^{j}-\right.\right.\right.$ $\left.\left.\left.1,2^{k-1-j}-1\right\}\right)\right)$. The underlying graph of $\vec{G}_{k, e}^{j}$, is a bipartite graph, by Lemma 3.1, with $2^{k-2}$ vertices on each of its partite sets and its edge set consists of the edges of jumps $2^{j-1}-1$ and $2^{k-j-2}-1$, from $X$ to $Y$, where $X=\left\{0,2, \ldots, 2^{k-1}-2\right\}$ and $Y=\left\{1,3, \ldots, 2^{k-1}-1\right\}$ (recall that an arc of distance $2^{\ell}-1$ in $\vec{G}_{k, e}^{j}$ will become an arc of jump $2^{\ell-1}-1$ in the bipartite structure of $\vec{G}_{k, e}^{j}$ ). Clearly, $\vec{G}_{k, e}^{j}$ consists of $2^{j-1}$ anti-directed cycles as $\operatorname{gcd}\left(2^{k-2}, 2^{j-1}-1+2^{k-2}-2^{k-j-2}+1\left(\bmod 2^{k-2}\right)\right)=2^{j-1}$, see Remark 3.1.

We have $a_{j}=2^{j}-1$ and $b_{j}=2^{k-1-j}-1$; let $x_{j}=2(j-1)$. First we exhibit $2^{j-1}$ disjoint anti-directed cycles $\vec{C}_{j, e}^{i}, 1 \leq i \leq 2^{j-1}$, in $\vec{G}_{k, e}^{j}$, to obtain the required number of anti-directed paths for $\vec{H}_{j, e}$.

In the following list of $2^{j-1}$ anti-directed cycles, the anti-directed cycle $\vec{C}_{j, e}^{i}, 2 \leq i \leq 2^{j-1}$ is obtained from $\vec{C}_{j, e}^{i-1}$ by subtracting $2 b_{j}$ from each of its corresponding vertices and $\vec{C}_{j, e}^{1}$ is explicitly described; in the vertices of the following anti-directed cycles, the addition is taken modulo $2^{k-1}$ and $x_{j}=2(j-1)$. We list $2^{j-1}$ anti-directed cycles and then we prove that they are mutually disjoint.

$$
\begin{aligned}
\vec{C}_{j, e}^{1}= & x_{j}\left(x_{j}+a_{j}\right)\left(x_{j}+a_{j}-b_{j}\right)\left(x_{j}+2 a_{j}-b_{j}\right)\left(x_{j}+2 a_{j}-2 b_{j}\right) \ldots\left(x_{j}+2 b_{j}-a_{j}\right)\left(x_{j}+b_{j}-a_{j}\right)\left(x_{j}+b_{j}\right) x_{j} \\
\vec{C}_{j, e}^{2}= & \left(x_{j}-2 b_{j}\right)\left(x_{j}+a_{j}-2 b_{j}\right)\left(x_{j}+a_{j}-3 b_{j}\right)\left(x_{j}+2 a_{j}-3 b_{j}\right)\left(x_{j}+2 a_{j}-4 b_{j}\right) \ldots,\left(x_{j}-a_{j}\right) \\
& \left(x_{j}-b_{j}-a_{j}\right)\left(x_{j}-b_{j}\right)\left(x_{j}-2 b_{j}\right) \\
\vec{C}_{j, e}^{3}= & \left(x_{j}-4 b_{j}\right)\left(x_{j}+a_{j}-4 b_{j}\right)\left(x_{j}+a_{j}-5 b_{j}\right)\left(x_{j}+2 a_{j}-5 b_{j}\right)\left(x_{j}+2 a_{j}-6 b_{j}\right) \ldots\left(x_{j}-2 b_{j}-a_{j}\right) \\
& \left(x_{j}-3 b_{j}-a_{j}\right)\left(x_{j}-3 b_{j}\right)\left(x_{j}-4 b_{j}\right) \\
\vdots & \vdots \\
\vec{C}_{j, e}^{i}= & \left(x_{j}-(i-1) 2 b_{j}\right)\left(x_{j}+a_{j}-(i-1) 2 b_{j}\right)\left(x_{j}+a_{j}-b_{j}-(i-1) 2 b_{j}\right)\left(x_{j}+2 a_{j}-b_{j}-(i-1) 2 b_{j}\right) \\
& \left(x_{j}+2 a_{j}-2 b_{j}-(i-1) 2 b_{j}\right) \ldots\left(x_{j}+2 b_{j}-a_{j}-(i-1) 2 b_{j}\right)\left(x_{j}+b_{j}-a_{j}-(i-1) 2 b_{j}\right)\left(x_{j}+b_{j}-(i-1) 2 b_{j}\right) \\
& \left(x_{j}-(i-1) 2 b_{j}\right) \\
\vdots & \vdots \\
\vec{C}_{j, e}^{2^{j-1}} & =
\end{aligned}
$$

In $\vec{G}_{k, e}^{j}$, the vertices $1,3,5, \ldots, 2^{k-1}-1$ are called the odd vertices and $0,2,4, \ldots, 2^{k-1}-2$ are called the even vertices. In $\vec{C}_{j, e}^{1}$, the odd vertices and even vertices alternate and an odd vertex is obtained by adding $a_{j}$ to its preceding (even) vertex, along the anti-directed cycle, and an even vertex is obtained from its preceding (odd) vertex by adding $\left(-b_{j}\right)$ to it. We obtain the required $2^{j-1}$ antidirected paths, denoted by $\vec{H}_{j, e}^{i}, 1 \leq i \leq 2^{j-1}$, from $\vec{C}_{j, e}^{i}, 1 \leq i \leq 2^{j-1}$, by deleting its last arc, namely, $\overrightarrow{\left(x_{j}-(i-1) 2 b_{j}\right)\left(x_{j}-(i-1) 2 b_{j}+b_{j}\right)}$. The origins of the $2^{j-1}$ anti-directed paths $\vec{H}_{j, e}^{i}, 1 \leq$ $i \leq 2^{j-1}$, are the origins of $\vec{C}_{j, e}^{i}$, namely, $x_{j}, x_{j}-2 b_{j}, x_{j}-4 b_{j}, \ldots, x_{j}-\left(2^{j}-2\right) b_{j}$. The union of 
these $2^{j-1}$ anti-directed paths $\vec{H}_{j, e}^{i}, 1 \leq i \leq 2^{j-1}$, is denoted by $\vec{H}_{j, e}$, that is, $\vec{H}_{j, e}=\bigcup_{i=1}^{2^{j-1}} \vec{H}_{j, e}^{i}$.

Next we prove that the vertices of these anti-directed paths $\vec{H}_{j, e}^{i}, 1 \leq i \leq 2^{j-1}$, form a partition of the vertex set of $\vec{G}_{k, e}^{j}$ (and hence that of $\vec{G}_{k, e}$ ).

Claim 1. The origins of the anti-directed paths $\vec{H}_{j, e}^{2}, \vec{H}_{j, e}^{3}, \ldots, \vec{H}_{j, e}^{2^{j-1}}$, namely, $\left(x_{j}-2 b_{j}\right),\left(x_{j}-\right.$ $\left.4 b_{j}\right),\left(x_{j}-6 b_{j}\right), \ldots,\left(x_{j}-\left(2^{j}-2\right) b_{j}\right)$ are all distinct from the even vertices of $\vec{H}_{j, e}^{1}$.

If $x_{j}-2 \ell b_{j}$, the origin of $\vec{H}_{j, e}^{\ell+1}$, is in $\vec{H}_{j, e}^{1}$, then, as $x_{j}-2 \ell b_{j}$ is even, it should be of the form $x_{j}+r a_{j}-r b_{j}$, for some $r \neq 0$ and $r \leq 2^{k-j-1}-1 ; r \leq 2^{k-j-1}-1$ follows from the fact that each antidirected cycle is of length $2^{k-j}$ and among the vertices of the anti-directed cycle, only half of them can be even vertices and further the origin is an even vertex. Hence $x_{j}-2 \ell b_{j} \equiv x_{j}+r a_{j}-r b_{j}\left(\bmod 2^{k-1}\right)$, that is,

$$
\begin{aligned}
r b_{j}-r a_{j}-2 \ell b_{j} & \equiv 0\left(\bmod 2^{k-1}\right) \\
r\left(b_{j}-a_{j}\right)-2 \ell b_{j} & \equiv 0\left(\bmod 2^{k-1}\right), \text { a contradiction, }
\end{aligned}
$$

for, if the congruence holds then, as $2^{j} \mid b_{j}-a_{j}$ and $2^{j} \mid 2^{k-1}$, we must have $2^{j} \mid 2 \ell b_{j}$, but this is not the case as $b_{j}$ is odd and $\ell \leq 2^{j-1}-1$. This implies that the origins of the anti-directed paths $\vec{H}_{j, e}^{2}, \vec{H}_{j, e}^{3}, \ldots, \vec{H}_{j, e}^{2^{j-1}}$ are all distinct from the even vertices of $\vec{H}_{j, e}^{1}$.

This completes the proof of Claim 1.

Claim 2. $\vec{H}_{j, e}^{\ell+1}$ and $\vec{H}_{j, e}^{\ell^{\prime}+1}, \ell \neq \ell^{\prime}$, are all disjoint.

It is enough to show that the set of even vertices of $\vec{H}_{j, e}^{\ell+1}$ and the even vertices of $\vec{H}_{j, e}^{\ell^{\prime}+1}$ are disjoint. Assume that $u$ is an even vertex common to both $\vec{H}_{j, e}^{\ell+1}$ and $\vec{H}_{j, e}^{\ell^{\prime}+1}$ for some $\ell^{\prime} \neq \ell$. Without loss of generality assume that $\ell^{\prime}<\ell$. As $u$ is an even vertex in $\vec{H}_{j, e}^{\ell+1}, u=x_{j}-2 \ell b_{j}+r\left(a_{j}-b_{j}\right)$ for some $r \leq 2^{k-j-1}-1$. As $u$ is also in $\vec{H}_{j, e}^{\ell^{\prime}+1}$, the vertex $x_{j}-2 \ell b_{j}$ must be in $\vec{H}_{j, e}^{\ell^{\prime}+1}$, since the difference between two even vertices is a multiple of $a_{j}-b_{j}$; since $x_{j}-2 \ell^{\prime} b_{j}$ is the origin of $\vec{H}_{j, e}^{\ell^{\prime}+1}$, both $x_{j}-2 \ell^{\prime} b_{j}$ and $x_{j}-2 \ell b_{j}$ are in $\vec{H}_{j, e}^{\ell^{\prime}+1}$ and they cannot be equal as $\ell \neq \ell^{\prime}$. If we add $2 \ell^{\prime} b_{j}$ to each vertex of $\vec{H}_{j, e}^{\ell^{\prime}+1}$, then the vertex $x_{j}-2\left(\ell-\ell^{\prime}\right) b_{j}$ is in $\vec{H}_{j, e}^{1}$, a contradiction to Claim 1 as $x_{j}-2\left(\ell-\ell^{\prime}\right) b_{j}$ is the origin of the anti-directed path $\vec{H}_{j, e}^{\ell-\ell^{\prime}+1}$.

This completes the proof of Claim 2.

Clearly, the arcs of $G_{k, e}^{j}$ which are not on the anti-directed paths $\vec{H}_{j, e}^{i}, 1 \leq i \leq 2^{j-1}$, are the $2^{j-1}$ $\operatorname{arcs} \overrightarrow{x_{j}\left(x_{j}+b_{j}\right)}, \overrightarrow{\left(x_{j}-2 b_{j}\right)\left(x_{j}-b_{j}\right)}, \overrightarrow{\left(x_{j}-4 b_{j}\right)\left(x_{j}-3 b_{j}\right)}, \ldots, \overrightarrow{\left(x_{j}-\left(2^{j}-2\right) b_{j}\right)\left(x_{j}-\left(2^{j}-3\right) b_{j}\right)}$, namely, the last arcs of the anti-directed cycles $\vec{C}_{j, e}^{i}, 1 \leq i \leq 2^{j-1}$.

As $\vec{G}_{k, e} \cong \vec{G}_{k, o}$, it can be verified that $\vec{G}_{k, e}^{j} \cong \vec{G}_{k, o}^{j}$. As $\vec{G}_{k, e}^{j}$ has $2^{j-1}$ disjoint anti-directed cycles, $\vec{G}_{k, o}^{j}$ also has $2^{j-1}$ disjoint anti-directed cycles. Similar to $\vec{C}_{j, e}^{i}, 1 \leq i \leq 2^{j-1}$, of $\vec{G}_{k, e}^{j}$, we obtain $2^{j-1}$ disjoint anti-directed cycles $\vec{C}_{j, o}^{i}, 1 \leq i \leq 2^{j-1}$, in $\vec{G}_{k, o}^{j}$.

Addition is taken modulo $2^{k-1}$ in the vertices of the following anti-directed cycles and in the following cycles also $x_{j}=2(j-1), a_{j}=2^{j}-1$ and $b_{j}=2^{k-j-1}-1$. 


$$
\begin{aligned}
\vec{C}_{j, o}^{1}=\quad & x_{j}\left(x_{j}-a_{j}\right)\left(x_{j}-a_{j}+b_{j}\right)\left(x_{j}-2 a_{j}+b_{j}\right)\left(x_{j}-2 a_{j}+2 b_{j}\right) \ldots\left(x_{j}-2 b_{j}+a_{j}\right)\left(x_{j}-b_{j}+a_{j}\right) \\
& \left(x_{j}-b_{j}\right) x_{j} \\
& \left(x_{j}-2 b_{j}\right)\left(x_{j}-a_{j}-2 b_{j}\right)\left(x_{j}-a_{j}-b_{j}\right)\left(x_{j}-2 a_{j}-b_{j}\right)\left(x_{j}-2 a_{j}\right) \ldots\left(x_{j}-4 b_{j}+a_{j}\right) \\
\vec{C}_{j, o}^{2}=\left(x_{j}-3 b_{j}+a_{j}\right)\left(x_{j}-3 b_{j}\right)\left(x_{j}-2 b_{j}\right) & \\
& \left(x_{j}-4 b_{j}\right)\left(x_{j}-a_{j}-4 b_{j}\right)\left(x_{j}-a_{j}-3 b_{j}\right)\left(x_{j}-2 a_{j}-3 b_{j}\right)\left(x_{j}-2 a_{j}-2 b_{j}\right) \ldots\left(x_{j}-6 b_{j}+a_{j}\right) \\
& \left(x_{j}-5 b_{j}+a_{j}\right)\left(x_{j}-5 b_{j}\right)\left(x_{j}-4 b_{j}\right) \\
\vec{C}_{j, o}^{3}= & \vdots \\
\vec{C}_{j, o}^{i}=\quad & \left(x_{j}-(i-1) 2 b_{j}\right)\left(x_{j}-a_{j}-(i-1) 2 b_{j}\right)\left(x_{j}-a_{j}+b_{j}-(i-1) 2 b_{j}\right)\left(x_{j}-2 a_{j}+b_{j}-(i-1) 2 b_{j}\right) \\
& \left(x_{j}-2 a_{j}+2 b_{j}-(i-1) 2 b_{j}\right) \ldots\left(x_{j}-2 b_{j}+a_{j}-(i-1) 2 b_{j}\right)\left(x_{j}-b_{j}+a_{j}-(i-1) 2 b_{j}\right) \\
& \left(x_{j}-b_{j}-(i-1) 2 b_{j}\right)\left(x_{j}-(i-1) 2 b_{j}\right) \\
\vec{C}_{j, o}^{2^{j-1}-1}= & \left(x_{j}-\left(2^{j}-4\right) b_{j}\right)\left(x_{j}-a_{j}-\left(2^{j}-4\right) b_{j}\right)\left(x_{j}-a_{j}-\left(2^{j}-5\right) b_{j}\right)\left(x_{j}-2 a_{j}-\left(2^{j}-5\right) b_{j}\right) \\
& \left(x_{j}-2 a_{j}-\left(2^{j}-6\right) b_{j}\right) \ldots,\left(x_{j}+a_{j}-\left(2^{j}-2\right) b_{j}\right)\left(x_{j}+a_{j}-\left(2^{j}-3\right) b_{j}\right)\left(x_{j}-\left(2^{j}-3\right) b_{j}\right) \\
& \left(x_{j}-\left(2^{j}-4\right) b_{j}\right) \\
& \left(x_{j}-\left(2^{j}-2\right) b_{j}\right)\left(x_{j}-a_{j}-\left(2^{j}-2\right) b_{j}\right)\left(x_{j}-a_{j}-\left(2^{j}-3\right) b_{j}\right)\left(x_{j}-2 a_{j}-\left(2^{j}-3\right) b_{j}\right) \\
\vec{C}_{j, o}^{2^{j-1}}=\left(x_{j}-2 a_{j}-\left(2^{j}-4\right) b_{j}\right) \ldots\left(x_{j}+a_{j}-2^{j} b_{j}\right)\left(x_{j}+a_{j}-\left(2^{j}-1\right) b_{j}\right)\left(x_{j}-\left(2^{j}-1\right) b_{j}\right) & \left(x_{j}-\left(2^{j}-2\right) b_{j}\right)
\end{aligned}
$$

The anti-directed cycle $\vec{C}_{j, o}^{i}, 2 \leq i \leq 2^{j-1}$, is obtained from $\vec{C}_{j, o}^{i-1}$ by subtracting $2 b_{j}$ from each of its vertices corresponding vertices and $\vec{C}_{j, o}^{1}$ is explicitly described.

We obtain $2^{j-1}$ anti-directed paths, denoted by $\vec{H}_{j, o}^{i}$, from $\vec{C}_{j, o}^{i}, 1 \leq i \leq 2^{j-1}$ by deleting its last arc, namely $\overrightarrow{\left(x_{j}-(i-1) 2 b_{j}-b_{j}\right)\left(x_{j}-(i-1) 2 b_{j}\right)}$. We assume that the origin of $\vec{H}_{j, o}^{i}, 1 \leq i \leq 2^{j-1}$, is the origin of $\vec{C}_{j, o}^{i}$; hence the origins are $x_{j}, x_{j}-2 b_{j}, x_{j}-4 b_{j}, \ldots, x_{j}-\left(2^{j}-2\right) b_{j}$.

Clearly the origin of the path $\vec{H}_{j, e}^{i}$ is same as the origin of $\vec{H}_{j, o}^{i}, 1 \leq i \leq 2^{j-1}$. Similarly the terminus of $\vec{H}_{j, o}^{i}$ is the terminus of $\vec{H}_{j, e}^{i+1}$ for all $i$ except for $i=2^{j-1}$. But we want to reconstruct $\vec{H}_{j, o}^{2^{j-1}}$, suitably, as an anti-directed path such that the reconstructed path and $\vec{H}_{j, e}^{1}$ have the same terminus.

Let $S_{j}$ and $T_{j}$ denote the sets of origins and termini of the anti-directed paths $\vec{H}_{j, e}^{i}, 1 \leq i \leq 2^{j-1}$, respectively.

For the reconstruction of $\vec{H}_{j, o}^{2^{j-1}}$, we need to prove that the vertices $x_{j}+a_{j}+b_{j}, x_{j}+b_{j}, x_{j}+2 b_{j}$ and $x_{j}+a_{j}+b_{j}+1$ are on $\vec{C}_{j, o}^{2^{j-1}}$ and the three vertices $x_{j}+a_{j}+b_{j}, x_{j}+b_{j}, x_{j}+2 b_{j}$ are consecutive vertices along $\vec{C}_{j, o}^{2^{j-1}}$. In $\vec{C}_{j, o}^{2^{j-1}}$, the vertex $\left(x_{j}-a_{j}-\left(2^{j}-2\right) b_{j}\right)$ is the immediate next vertex of the origin $\left(x_{j}-\left(2^{j}-2\right) b_{j}\right)$. Further, $\left(x_{j}-a_{j}-\left(2^{j}-2\right) b_{j}\right) \equiv x_{j}+2 b_{j}+1\left(\bmod 2^{k-1}\right)$, 
since $a_{j}=2^{j}-1, b_{j}=2^{k-j-1}-1$,

$$
\begin{aligned}
-a_{j}-\left(2^{j}-2\right) b_{j} & =-\left(2^{j}-1\right)-\left(2^{j}-2\right)\left(2^{k-1-j}-1\right) \\
& =1-2^{j} \cdot 2^{k-1-j}+2.2^{k-1-j}-2 \\
& =-2^{k-1}+2.2^{k-1-j}-1 \\
& \equiv 2 \cdot\left(2^{k-1-j}-1\right)+2-1\left(\bmod 2^{k-1}\right) \\
& \equiv 2 b_{j}+1\left(\bmod 2^{k-1}\right)
\end{aligned}
$$

Consequently, $x_{j}-a_{j}-\left(2^{j}-2\right) b_{j} \equiv x_{j}+2 b_{j}+1\left(\bmod 2^{k-1}\right)$. Often we recall this congruence $x_{j}+2 b_{j}+1 \equiv x_{j}-a_{j}-\left(2^{j}-2\right) b_{j}\left(\bmod 2^{k-1}\right)$ in the future. In many places we write $x_{j}+2 b_{j}+1$ instead of $x_{j}-a_{j}-\left(2^{j}-2\right) b_{j}$.

It is enough to show that $x_{j}+b_{j}$ is in $\vec{C}_{j, o}^{2^{j-1}}$, since by adding $b_{j}$ (resp. $a_{j}$ ) to an odd vertex we get its succeeding (resp. preceding) vertex, namely $x_{j}+2 b_{j}$ (resp. $x_{j}+a_{j}+b_{j}$ ), in the anti-directed cycle. Now we prove that $x_{j}+b_{j}$ is in $\vec{C}_{j, o}^{2 j-1}$. Clearly, $b_{j}-a_{j} \equiv 0\left(\bmod 2^{j}\right)$ as $j \leq\left\lfloor\frac{k}{2}\right\rfloor-1 ;$ let $b_{j}-a_{j}=m 2^{j}$, where $m$ is odd.

$$
\begin{aligned}
\text { Now } a_{j} b_{j}+a_{j}+b_{j}+1 & =\left(a_{j}+1\right)\left(b_{j}+1\right) \\
& =\left(2^{j}\right)\left(2^{k-j-1}\right) \\
& =2^{k-1} \equiv 0\left(\bmod 2^{k-1}\right)
\end{aligned}
$$

Hence

$$
\begin{aligned}
b_{j}+2^{j} & =b_{j}+a_{j}+1 \\
& \equiv-a_{j} b_{j}\left(\bmod 2^{k-1}\right)
\end{aligned}
$$

As $x_{j}$ is even, $x_{j}+b_{j}$ and the last vertex $x_{j}-\left(2^{j}-1\right) b_{j}=x_{j}-a_{j} b_{j}$ of the anti-directed cycle $\vec{C}_{j, o}^{2^{j-1}}$ are odd. Hence the vertex $x_{j}+b_{j}$ is in $\vec{C}_{j, o}^{2^{j-1}}$ if and only if $x_{j}+b_{j}+\ell\left(b_{j}-a_{j}\right) \equiv x_{j}-a_{j} b_{j}\left(\bmod 2^{k-1}\right)$, for some $\ell<2^{k-j-1}$, since the difference between two odd vertices along $\vec{C}_{j, o}^{2^{j-1}}$ is a multiple of $b_{j}-a_{j}$ and there can be only $2^{k-j-1}$ odd vertices in an anti-directed cycles.

$$
\begin{aligned}
x_{j}+b_{j} \text { is in } \vec{C}_{j, o}^{2^{j-1}} & \\
\Longleftrightarrow x_{j}+b_{j}+\ell\left(b_{j}-a_{j}\right) & \equiv x_{j}-a_{j} b_{j}\left(\bmod 2^{k-1}\right) \\
\Longleftrightarrow b_{j}+\ell\left(b_{j}-a_{j}\right) & \equiv-a_{j} b_{j}\left(\bmod 2^{k-1}\right) \\
\Longleftrightarrow b_{j}+\ell m 2^{j} & \equiv-a_{j} b_{j}\left(\bmod 2^{k-1}\right), \text { since } b_{j}-a_{j}=m 2^{j} . \\
\Longleftrightarrow b_{j}+\ell m 2^{j} & \equiv b_{j}+2^{j}\left(\bmod 2^{k-1}\right), \text { by }(2) \\
\Longleftrightarrow \ell m 2^{j} & \equiv 2^{j}\left(\bmod 2^{k-1}\right) \\
\Longleftrightarrow \ell m & \equiv 1\left(\bmod 2^{k-j-1}\right)
\end{aligned}
$$

As $b_{j}-a_{j}=m 2^{j}$ and $b_{j}-a_{j}$ are known, $m$ is known. Thus (3) gives that $x_{j}+b_{j}$ is in $\vec{C}_{j, o}^{2^{j-1}}$ if and only if the congruence in (3) has a solution. As $m$ is odd and $\operatorname{gcd}\left(m, 2^{k-j-1}\right)=1$, the congruence in (3) has a unique solution. Thus $x_{j}+b_{j}$ must be in $\vec{C}_{j, o}^{2^{j-1}}$. 


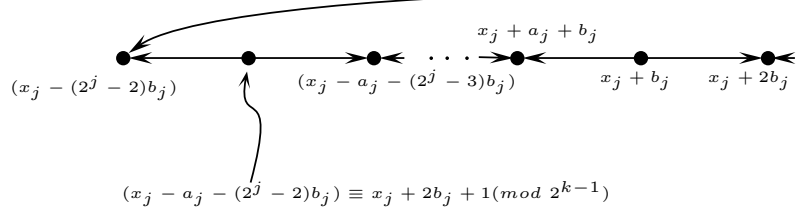

$(a)$

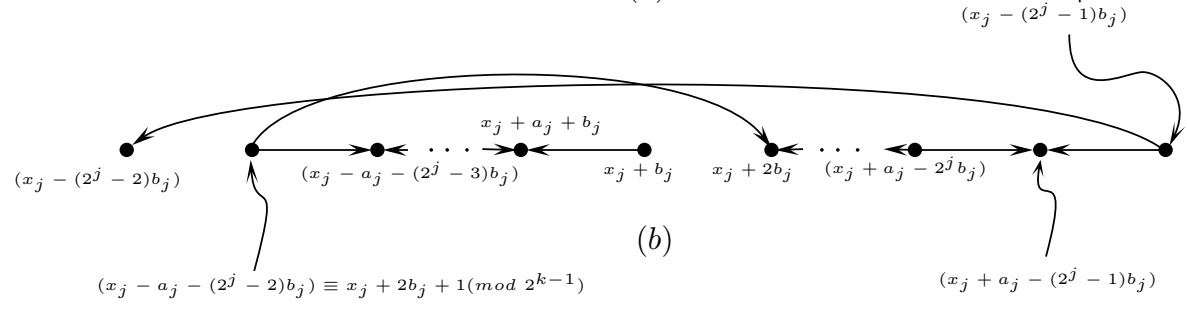

Fig. 6: Figure 6(a) shows the cycle $\vec{C}_{j, o}^{2^{j-1}}$ as in (4) above and Figure 6(b) shows the reconstructed anti-directed path $\vec{H}_{j, o}^{2^{j-1}}$ with origin $x_{j}-\left(2^{j}-2\right) b_{j}$ and terminus $x_{j}+b_{j}$.

Clearly, the vertex next to $x_{j}+b_{j}$ along $\vec{C}_{j, o}^{2^{j-1}}$ is $x_{j}+2 b_{j}$; therefore, $\vec{C}_{j, o}^{2^{j-1}}$ must be of the form

$$
\begin{array}{r}
\vec{C}_{j, o}^{2^{j-1}}=\left(x_{j}-\left(2^{j}-2\right) b_{j}\right)\left(x_{j}-a_{j}-\left(2^{j}-2\right) b_{j}\right)\left(x_{j}-a_{j}-\left(2^{j}-3\right) b_{j}\right)\left(x_{j}-2 a_{j}-\left(2^{j}-3\right) b_{j}\right) \\
\left(x_{j}-2 a_{j}-\left(2^{j}-4\right) b_{j}\right) \ldots,\left(x_{j}+a_{j}+b_{j}\right)\left(x_{j}+b_{j}\right)\left(x_{j}+2 b_{j}\right)\left(x_{j}+2 b_{j}-a_{j}\right) \ldots\left(x_{j}+a_{j}-2^{j} b_{j}\right) \\
\left(x_{j}+a_{j}-\left(2^{j}-1\right) b_{j}\right)\left(x_{j}-\left(2^{j}-1\right) b_{j}\right)\left(x_{j}-\left(2^{j}-2\right) b_{j}\right)
\end{array}
$$

Hence the reconstructed last anti-directed path $\vec{H}_{j, o}^{2^{j-1}}$ is obtained from (4) by deleting the first arc $\overrightarrow{\left(x_{j}-a_{j}-\left(2^{j}-2\right) b_{j}\right)\left(x_{j}-\left(2^{j}-2\right) b_{j}\right)}$ and the arc $\overrightarrow{\left(x_{j}+b_{j}\right)\left(x_{j}+2 b_{j}\right)}$ and, adding the arc $\overrightarrow{\left(x_{j}-a_{j}-\left(2^{j}-2\right) b_{j}\right)\left(x_{j}+2 b_{j}\right)}$; recall that $\left(x_{j}-a_{j}-\left(2^{j}-2\right) b_{j}\right) \equiv\left(x_{j}+2 b_{j}+1\right)\left(\bmod 2^{k-1}\right)$; see Figure 6. In fact, the arc $\overline{\left(x_{j}+2 b_{j}+1\right)\left(x_{j}+2 b_{j}\right)}$ is never used in the previous anti-directed paths $\vec{H}_{j, o}^{i}, 1 \leq i \leq 2^{j-1}-1$, as it is an arc of distance $2^{k-1}-1$ in $\vec{G}_{k}$, which is neither in $\vec{G}_{k, e}^{j}$ nor in $\vec{G}_{k, o}^{j}, 1 \leq j \leq\left\lfloor\frac{k}{2}\right\rfloor-1$, see Figure 6 , that is,

$$
\begin{array}{r}
\vec{H}_{j, o}^{2^{j-1}}=\left(x_{j}-\left(2^{j}-2\right) b_{j}\right)\left(x_{j}-\left(2^{j}-1\right) b_{j}\right)\left(x_{j}+a_{j}-\left(2^{j}-1\right) b_{j}\right)\left(x_{j}+a_{j}-2^{j} b_{j}\right) \ldots \\
\left(x_{j}+2 b_{j}\right)\left(x_{j}-a_{j}-\left(2^{j}-2\right) b_{j}\right)\left(x_{j}-a_{j}-\left(2^{j}-3\right) b_{j}\right)\left(x_{j}-2 a_{j}-\left(2^{j}-3\right) b_{j}\right) \\
\left(x_{j}-2 a_{j}-\left(2^{j}-4\right) b_{j}\right) \ldots,\left(x_{j}+b_{j}\right)
\end{array}
$$

In (5) note that the vertex $\left(x_{j}-a_{j}-\left(2^{j}-2\right) b_{j}\right) \equiv\left(x_{j}+2 b_{j}+1\right)\left(\bmod 2^{k-1}\right)$. For all our future reference $\vec{H}_{j, o}^{j-1}$ will refer to the reconstructed anti-directed path in (5).

This path contains the arc $\overrightarrow{\left(x_{j}+2 b_{j}+1\right)\left(x_{j}+2 b_{j}\right)}$ which is an arc of distance $2^{k-1}-1$ of $\vec{G}_{k}$. Consequently, the arcs of $\vec{G}_{k, o}^{j}$ which are not on the anti-directed paths $\vec{H}_{j, o}^{i}, 1 \leq i \leq 2^{j-1}$ are the $2^{j-1}+1 \operatorname{arcs}$ 
$\overrightarrow{\left(x_{j}-b_{j}\right) x_{j}}, \overrightarrow{\left(x_{j}-3 b_{j}\right)\left(x_{j}-2 b_{j}\right)}, \overrightarrow{\left(x_{j}-5 b_{j}\right)\left(x_{j}-4 b_{j}\right)}, \ldots, \overrightarrow{\left(x_{j}-\left(2^{j}-3\right) b_{j}\right)\left(x_{j}-\left(2^{j}-4\right) b_{j}\right)}$, $\overrightarrow{\left(x_{j}+b_{j}\right)\left(x_{j}+2 b_{j}\right)}, \overrightarrow{\left(x_{j}-a_{j}-\left(2^{j}-2\right) b_{j}\right)\left(x_{j}-\left(2^{j}-2\right) b_{j}\right)}$, that is, these are the last arcs of the $2^{j-1}-1$ anti-directed cycles $\vec{C}_{j, o}^{i}, 1 \leq i \leq 2^{j-1}-1$, and the two arcs deleted from $\vec{C}_{j, o}^{2^{j-1}}$ to obtain the reconstructed $\vec{H}_{j, o}^{2^{j-1}}$.

Let the union of these $2^{j-1}$ anti-directed paths $\vec{H}_{j, o}^{i}, 1 \leq i \leq 2^{j-1}$, be denoted by $\vec{H}_{j, o}$, that is, $\vec{H}_{j, o}=\bigcup_{i=1}^{2^{j-1}} \vec{H}_{j, o}^{i}$.

Thus for each pair of distances $\left(a_{j}=2^{j}-1, b_{j}=2^{k-j-1}-1\right)$ of $\vec{G}_{k}$ and $x_{j}=2(j-1)$, we have the pair of digraphs $\vec{H}_{j, e}$ and $\vec{H}_{j, o}, 1 \leq j \leq\left\lfloor\frac{k}{2}\right\rfloor-1$.

Recall that $S_{j}$ and $T_{j}$ denote the origins and termini of the anti-directed paths $\vec{H}_{j, e}^{i}, 1 \leq i \leq 2^{j-1}$, respectively, and also they are the origins and termini of the anti-directed paths of $\vec{H}_{j, o}^{i}$. Let $A_{j}=S_{j} \cup T_{j}$ and set $t=\left\lfloor\frac{k}{2}\right\rfloor-1$.

Next we prove that $A_{j}, 1 \leq j \leq\left\lfloor\frac{k}{2}\right\rfloor-1$ are mutually disjoint. We know that the origin and terminus of $\vec{H}_{j, e}^{i}, 1 \leq i \leq 2^{j-1}$, are $x_{j}-(i-1) 2 b_{j}$ and $x_{j}-(i-1) 2 b_{j}+b_{j}\left(=x_{j}-(2 i-3) b_{j}\right)$, respectively, and they are independent of $a_{j}$.

By our choice $x_{j}=2(j-1), x_{j-1}=2(j-1-1)=2(j-1)-2=x_{j}-2$; thus, recursively we can write $x_{\ell}$ in terms of $x_{j}$ for all $\ell<j$. Further, as $a_{j}=2^{j}-1, a_{j-1}=2^{j-1}-1=\frac{2^{j}-2}{2}=\frac{a_{j}-1}{2}$ and $b_{j}=$ $2^{k-j-1}-1$ implies $b_{j-1}=2^{k-j}-1=2.2^{k-j-1}-1=2\left(2^{k-j-1}-1\right)+1=2 b_{j}+1$. Thus, recursively we can write both $a_{\ell}$ and $b_{\ell}$ in terms of $a_{j}$ and $b_{j}$, respectively, for all $\ell<j$. Hence all $x_{j}$ 's $b_{j}$ 's and $a_{j}$ 's can be written in terms of $x_{t}, b_{t}$ and $a_{t}$, respectively, where $t=\left\lfloor\frac{k}{2}\right\rfloor-1$. If $j=t-r$ for some $r>0$, then $x_{j}=x_{t-r}=x_{t-r+1}-2$. Again $x_{t-r+1}=x_{t-r+2}-2$, thus $x_{j}=x_{t-r}=x_{t-r+1}-2=x_{t-r+2}-4$. Proceeding like this, we get $x_{j}=x_{t-r}=x_{t}-2 r$. Similarly for $j=t-r$, recursively applying the above relation, we get $b_{j}=b_{t-r}=2 b_{t-r+1}+1=2\left(2 b_{t-r+2}+1\right)+1=4 b_{t-r+2}+3=4\left(2 b_{t-r+3}+1\right)+3=$ $8 b_{t-r+3}+7$, etc. Proceeding like this, we get $b_{j}=b_{t-r}=2^{r} b_{t}+2^{r}-1$. Also for $j=t-r$, recursively applying the above relation, we get $a_{j}=a_{t-r}=\frac{a_{t-r+1}-1}{2}=\frac{\frac{a_{t-r+2}-1}{2}-1}{2}=\frac{a_{t-r+2}-3}{4}=\frac{\frac{a_{t-r+3}-1}{2}-3}{4}=$ $\frac{a_{t-r+3}-7}{8}=e t c$. Proceeding like this, we get $a_{j}=a_{t-r}=\frac{a_{t}-2^{r}+1}{2^{r}}$.

For our convenience, we shall denote the origin and terminus of an anti-directed path $\vec{H}_{j, e}^{\ell}$ by the ordered pair $(r, s)$ where $r$ is the origin and $s$ is the terminus of $\vec{H}_{j, e}^{\ell}$, respectively. For $t=\left\lfloor\frac{k}{2}\right\rfloor-1$, by the above notation, the ordered pairs $\left(x_{t}, x_{t}+b_{t}\right),\left(x_{t}-2 b_{t}, x_{t}-b_{t}\right),\left(x_{t}-4 b_{t}, x_{t}-3 b_{t}\right),\left(x_{t}-6 b_{t}, x_{t}-\right.$ $\left.5 b_{t}\right), \ldots\left(x_{t}-\left(2^{t}-4\right) b_{t}, x_{t}-\left(2^{t}-5\right) b_{t}\right),\left(x_{t}-\left(2^{t}-2\right) b_{t}, x_{t}-\left(2^{t}-3\right) b_{t}\right)$ denote the origins and termini of the anti-directed paths $\vec{H}_{t, e}^{i}, 1 \leq i \leq 2^{t-1}$, in $\vec{H}_{t, e}$. Similarly, $\left(x_{t-1}, x_{t-1}+b_{t-1}\right),\left(x_{t-1}-\right.$ $\left.2 b_{t-1}, x_{t-1}-b_{t-1}\right),\left(x_{t-1}-4 b_{t-1}, x_{t-1}-3 b_{t-1}\right),\left(x_{t-1}-6 b_{t-1}, x_{t-1}-5 b_{t-1}\right), \ldots\left(x_{t-1}-\left(2^{t-1}-\right.\right.$ 4) $\left.b_{t-1}, x_{t-1}-\left(2^{t-1}-5\right) b_{t-1}\right),\left(x_{t-1}-\left(2^{t-1}-2\right) b_{t-1}, x_{t-1}-\left(2^{t-1}-3\right) b_{t-1}\right)$ denote the origins and termini of the anti-directed paths $\vec{H}_{t-1, e}^{i}, 1 \leq i \leq 2^{t-2}$, in $\vec{H}_{t-1, e}$. But we have seen above that $b_{t-1}=2 b_{t}+1$ and $x_{t-1}=x_{t}-2$. If we write $x_{t-1}$ and $b_{t-1}$ in terms of $x_{t}$ and $b_{t}$ to the origins and termini of the anti-directed paths of $\vec{H}_{t-1, e}$, the origins and termini of the anti-directed paths in $\vec{H}_{t-1, e}$ are $\left(x_{t}-2, x_{t}+2 b_{t}-1\right),\left(x_{t}-4 b_{t}-4, x_{t}-2 b_{t}-3\right),\left(x_{t}-8 b_{t}-6, x_{t}-6 b_{t}-5\right),\left(x_{t}-12 b_{t}-\right.$ $\left.8, x_{t}-10 b_{t}-7\right), \ldots,\left(x_{t}-\left(2^{t}-8\right) b_{t}-2^{t-1}+2, x_{t}-\left(2^{t}-10\right) b_{t}-2^{t-1}+3\right),\left(x_{t}-\left(2^{t}-4\right) b_{t}-\right.$ $\left.2^{t-1}, x_{t}-\left(2^{t}-6\right) b_{t}-2^{t-1}+1\right)$. Proceeding as above, the origin and terminal vertices of each of the anti-direted paths of $\vec{H}_{j, e}$ can be given in terms of $x_{t}$ and $b_{t}$. 


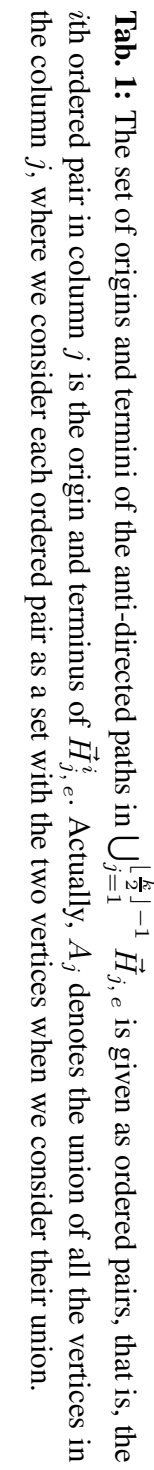

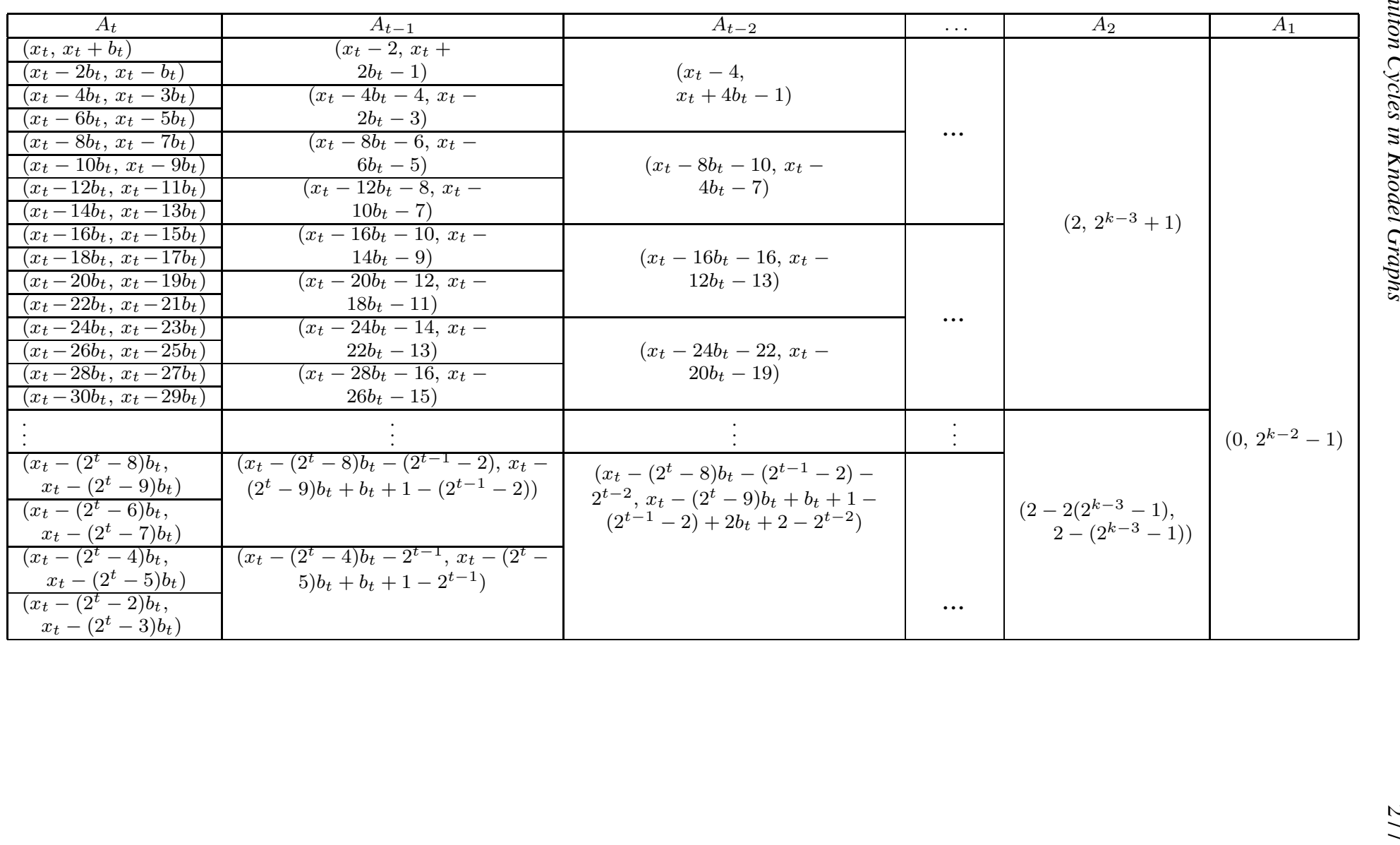


In general, the origins and termini of anti-directed paths of $\vec{H}_{j, e}$ are $\left(x_{j}, x_{j}+b_{j}\right),\left(x_{j}-2 b_{j}, x_{j}-\right.$ $\left.b_{j}\right),\left(x_{j}-4 b_{j}, x_{j}-3 b_{j}\right), \ldots,\left(x_{j}-(i-1) 2 b_{j}, x_{j}-(2 i-3) b_{j}\right), \ldots,\left(x_{j}-\left(2^{j}-2\right) b_{j}, x_{j}-\left(2^{j}-3\right) b_{j}\right)$. If $j=t-r$, then by the relation obtained above, $x_{j}=x_{t}-2 r$ and $b_{j}=2^{r} b_{t}+2^{r}-1$. Thus the origins and termini of anti-directed paths of $\vec{H}_{j, e}$ in terms of $x_{t}$ and $b_{t}$ are given by the ordered pairs $\left(x_{t}-2 r, x_{t}-2 r+2^{r} b_{t}+2^{r}-1\right),\left(x_{t}-2 r-2\left(2^{r} b_{t}+2^{r}-1\right), x_{t}-2 r-\left(2^{r} b_{t}+2^{r}-1\right)\right),\left(x_{t}-\right.$ $\left.2 r-4\left(2^{r} b_{t}+2^{r}-1\right), x_{t}-2 r-3\left(2^{r} b_{t}+2^{r}-1\right)\right), \ldots,\left(x_{t}-2 r-(i-1) 2\left(2^{r} b_{t}+2^{r}-1\right), x_{t}-2 r-\right.$ $\left.(2 i-3)\left(2^{r} b_{t}+2^{r}-1\right)\right), \ldots,\left(x_{t}-2 r-\left(2^{j}-2\right)\left(2^{r} b_{t}+2^{r}-1\right), x_{t}-2 r-\left(2^{j}-3\right)\left(2^{r} b_{t}+2^{r}-1\right)\right)$. Hereafter we shall represent the elements of $A_{j}$ in terms of $x_{t}$ and $b_{t}$.

We list the elements of $A_{j}, 1 \leq j \leq\left\lfloor\frac{k}{2}\right\rfloor-1$, in terms of $x_{t}$ and $b_{t}$, in ordered pairs in the Table 1 below with the elements of $A_{t}$ appearing in the first column, the elements of $A_{t-1}$ in the second column etc., and the last column contains the elements of $A_{1}$.

Next we shall prove that $A_{i} \cap A_{j}=\phi, i \neq j$; the intersection here is the intersection of elements of $A_{i}$ and $A_{j}$ (but not as intersection of ordered pairs of $A_{i}$ and $A_{j}$ described in the Table 1). For this it is enough to prove that the even vertices in the Table 1 are distinct. For, let $i<j$ with $i=t-s$ and $j=t-r$; hence $r<s$. By the above recursive relations we get $x_{j}=x_{t}-2 r, x_{i}=x_{t}-2 s, b_{j}=2^{r} b_{t}+2^{r}-1$ and $b_{i}=2^{s} b_{t}+2^{s}-1$. Suppose the origins in the $(\ell+1)$ th pair of $A_{j}$ in Table 1 , that is, $x_{j}-2 \ell b_{j}$ and the $\left(\ell^{\prime}+1\right)$ th pair of $A_{i}$, that is, $x_{i}-2 \ell^{\prime} b_{i}$ are same then

$$
\begin{aligned}
x_{j}-2 \ell b_{j} & \equiv x_{i}-2 \ell^{\prime} b_{i}\left(\bmod 2^{k-1}\right) ; \text { this implies } \\
x_{t}-2 r-2 \ell\left(2^{r} b_{t}+2^{r}-1\right) & \equiv x_{t}-2 s-2 \ell^{\prime}\left(2^{s} b_{t}+2^{s}-1\right)\left(\bmod 2^{k-1}\right)
\end{aligned}
$$

that is, $2 r+2^{r+1} \ell b_{t}+2^{r+1} \ell-2 \ell \equiv 2 s+2^{s+1} \ell^{\prime} b_{t}+2^{s+1} \ell^{\prime}-2 \ell^{\prime}\left(\bmod 2^{k-1}\right)$

that is, $2(r-s)+2^{r+1} \ell\left(b_{t}+1\right)-2 \ell \equiv 2^{s+1} \ell^{\prime}\left(b_{t}+1\right)-2 \ell^{\prime}\left(\bmod 2^{k-1}\right)$

$$
\begin{aligned}
\text { that is, } 2(r-s)+2\left(\ell^{\prime}-\ell\right) & \equiv 2^{s+1} \ell^{\prime} 2^{k-t-1}-2^{r+1} \ell 2^{k-t-1}\left(\bmod 2^{k-1}\right) \\
& \equiv A 2^{r+k-t}\left(\bmod 2^{k-1}\right) \text { for some constant } A \text {, that is, } \\
A 2^{r+k-t}-2\left(r-s+\ell^{\prime}-\ell\right) & \equiv 0\left(\bmod 2^{k-1}\right)
\end{aligned}
$$

However, we shall show that this congruence does not hold. We assume that the congurence holds and we obtain a contradicition. Observe that as $0 \leq r<s \leq\left\lfloor\frac{k}{2}\right\rfloor-1$ and $t=\left\lfloor\frac{k}{2}\right\rfloor-1$, we have $r+k-t \leq\left\lfloor\frac{k}{2}\right\rfloor-2+k-\left\lfloor\frac{k}{2}\right\rfloor+1=k-1$.

First we suppose that $A$ is odd. Since $A$ is odd, as $2^{r+k-t}$ divides both $A 2^{r+k-t}$ and $2^{k-1}$, then it must divide $2\left[r-s+\ell^{\prime}-\ell\right]$, that is, $2\left[r-s+\ell^{\prime}-\ell\right] \equiv 0\left(\bmod 2^{r+k-t}\right)$, which is a contradiction, as $0 \leq r<s \leq\left\lfloor\frac{k}{2}\right\rfloor-1,0 \leq \ell \leq 2^{t-r-1}-1$ and $0 \leq \ell^{\prime} \leq 2^{t-s-1}-1$.

Next we suppose that $A$ is even. Let $d$ be the maximum integer such that $2^{d} \mid A 2^{r+k-t}$. Now we consider the case $d \leq k-1$; then the proof follows similar to the case when $A$ is odd by replacing $r+k-t$ by $d$. Next we assume that $d>k-1$. Since $d>k-1,2^{k-1} \mid 2^{d}$. Then $2^{k-1}$ divides $2\left[r-s+\ell^{\prime}-\ell\right]$, that is, $2\left[r-s+\ell^{\prime}-\ell\right] \equiv 0\left(\bmod 2^{k-1}\right)$, which is a contradiction, as $0 \leq r<s \leq\left\lfloor\frac{k}{2}\right\rfloor-1,0 \leq \ell \leq 2^{t-r-1}-1$ and $0 \leq \ell^{\prime} \leq 2^{t-s-1}-1$.

Hence irrespective of the parity of $A$, the congruence does not hold. Thus the origins, that is, the even vertices of the ordered pairs listed in the columns $A_{i}$ and $A_{j}, i \neq j$, of Table 1 , are distinct and hence the odd vertices of the ordered pairs listed in the columns $A_{i}$ and $A_{j}, i \neq j$, of Table 1 are also distinct.

This completes the proof of the lemma. 
Lemma 3.3 There exist $\left\lfloor\frac{k}{2}\right\rfloor-1$ arc disjoint 2-diregular bieulerian digraphs $\vec{H}_{j}, 1 \leq j \leq\left\lfloor\frac{k}{2}\right\rfloor-1$, each of which is a spanning subdigraph of $\vec{G}_{k}^{\prime}$.

Proof: By Lemma 3.2, there exist $\left\lfloor\frac{k}{2}\right\rfloor-1$ arc disjoint spanning subdigraphs $\vec{H}_{j, e}$ and $\vec{H}_{j, o}, 1 \leq j \leq$ $\left\lfloor\frac{k}{2}\right\rfloor-1$, of $\vec{G}_{k, e}$ and $\vec{G}_{k, o}$, respectively. Clearly, each of the digraphs $\vec{H}_{j, e}$ and $\vec{H}_{j, o}$ consists of union of $2^{j-1}$ anti-directed paths with their origins and termini at $A_{j}=S_{j} \cup T_{j}$.

Next we construct $\left\lfloor\frac{k}{2}\right\rfloor-1$ spanning 2-diregular bieulerian digraphs using $\vec{H}_{j, e}$ and $\vec{H}_{j, o}, 1 \leq j \leq$ $\left\lfloor\frac{k}{2}\right\rfloor-1$. For a fixed $j$, we construct a spanning 2-diregular bieulerian digraph from $\vec{H}_{j, e}$ and $\vec{H}_{j, o}$ by

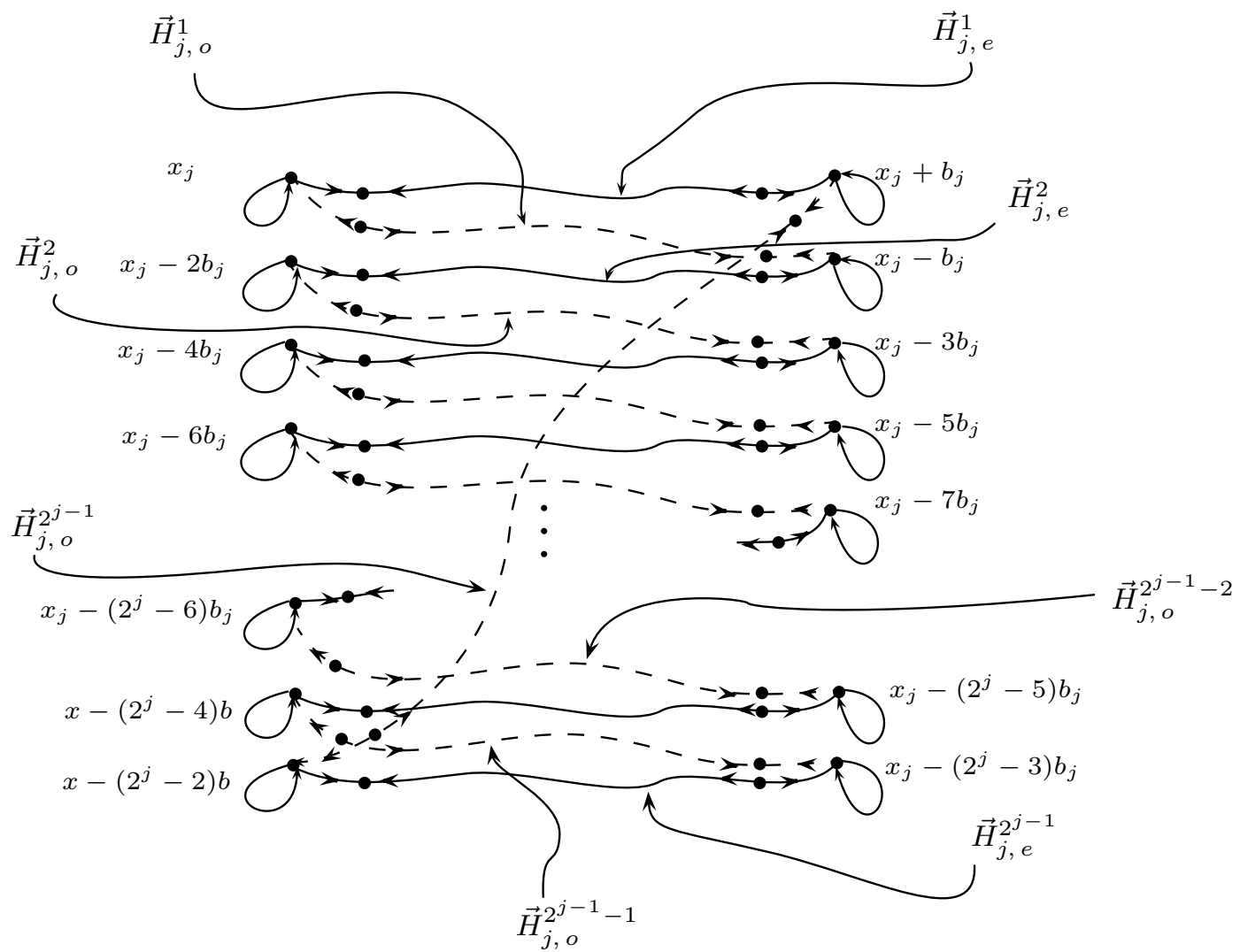

The anti-directed paths $\vec{H}_{j, e}^{i}, 1 \leq i \leq 2^{j-1}$, of $\vec{H}_{j, e}$ are shown in solid lines and the anti-directed paths $\vec{H}_{j, o}^{i}, 1 \leq$ $i \leq 2^{j-1}$, of $\vec{H}_{j, o}$ are shown in broken lines. For clarity of the figure, $\vec{H}_{j, o}^{i}$ is shown as vertex disjoint from $\vec{H}_{j, e}^{i}$, but it is not the case as both $\vec{H}_{j, e}$ and $\vec{H}_{j, o}$ are spanning subdigraphs of $\vec{G}_{k}$.

Fig. 7: 2-diregular spanning bieulerian digraph $\vec{H}_{j}$ constructed from $\vec{H}_{j, e}$ and $\vec{H}_{j, o}$ and loops added at each of the vertices of $A_{j}$. 
concatenating the anti-directed paths of them appropriately and adding some appropriate loops to it. That is, the spanning bieulerian digraph is $H_{j, e}^{1} \cup H_{j, o}^{2^{j-1}} \cup H_{j, e}^{2^{j-1}} \cup H_{j, o}^{2^{j-1}-1} \cup H_{j, e}^{2^{j-1}-1} \cup H_{j, o}^{2^{j-1}-2} \cup H_{j, e}^{2^{j-1}-2} \cup$ $H_{j, o}^{2^{j-1}-3} \cup H_{j, e}^{2^{j-1}-3} \cup \ldots \cup H_{j, o}^{2} \cup H_{j, e}^{2} \cup H_{j, o}^{1} \cup\left\{\right.$ directed loops at each of the vertices of $A_{j}$, see Figure 7. We denote the graph $\vec{H}_{j, e} \cup \vec{H}_{j, o}$ together with directed loops added at the vertices of $A_{j}$ by $\vec{H}_{j}$, see Figure 7. Clearly, $\vec{H}_{j}$ is eulerian and, $\vec{H}_{j}$ is aneulerian follows by moving along the anti-directed paths of $\vec{H}_{j, e}$ and $\vec{H}_{j, o}$, alternately, and the directed loops at the vertices of $A_{j}$; while obtaining an aneulerian tour, as and when a directed loop of $A_{j}$ is encountered when we visit through $\vec{H}_{j, e}$ or $\vec{H}_{j, o}$, the directed loop is visited in the clockwise or anticlockwise direction according to the requirement for the existence of an aneulerian tour.

This completes the proof of the lemma.

Next we prove that $W_{k, 2^{k}}, k \geq 6$, has $\left\lfloor\frac{k}{2}\right\rfloor-1$ edge disjoint Hamilton cycles.

Theorem 3.2 $W_{k, 2^{k}}$ contains $\left\lfloor\frac{k}{2}\right\rfloor-1$ edge disjoint Hamilton cycles.

Proof: By Lemma 3.3, $\vec{G}_{k}^{\prime}$ has $\left\lfloor\frac{k}{2}\right\rfloor-1$ arc disjoint 2-diregular spanning bieulerian subdigraphs $\vec{H}_{j}, 1 \leq$ $j \leq\left\lfloor\frac{k}{2}\right\rfloor-1$. Let $\vec{H}=\bigcup_{j=1}^{\left\lfloor\frac{k}{2}\right\rfloor-1} \vec{H}_{j}$. Now $\vec{H} \times \vec{K}_{2}=\bigoplus_{j=1}^{\left\lfloor\frac{k}{2}\right\rfloor-1}\left(\vec{H}_{j} \times \vec{K}_{2}\right)$. But the underlying graph of $\vec{H}_{j} \times \vec{K}_{2}, 1 \leq j \leq\left\lfloor\frac{k}{2}\right\rfloor-1$, is a Hamilton cycle, by Theorem 2.2. Thus the underlying graph of $\vec{H} \times \vec{K}_{2}$ is Hamilton cycle decomposable. As $\vec{H}$ is a spanning subdigraph of $\vec{G}_{k}^{\prime}$ and the underlying graph of $\vec{G}_{k}^{\prime} \times \vec{K}_{2}$ is isomorphic to $W_{k, 2^{k}}, W_{k, 2^{k}}$ contains $\left\lfloor\frac{k}{2}\right\rfloor-1$ edge disjoint Hamilton cycles.

To prove the existence of a Hamilton cycle decomposition of $W_{k, 2^{k}}$, it is enough to prove that $\vec{G}_{k}^{\prime \prime}=$ $\vec{G}_{k}^{\prime}-A(\vec{H})$, when $k$ is even or, $\vec{G}_{k}^{\prime \prime}=\vec{G}_{k}^{\prime}-\{A(\vec{H}) \cup \vec{F}\}$, where $\vec{F}$ stands for the set of arcs of distance $\frac{k+1}{2}$ of $\vec{G}_{k}^{\prime}$, when $k$ is odd, is a bieulerian digraph. If $\vec{G}_{k}^{\prime \prime}$ is bieulerian, then by Theorem 2.2, the underlying graph of $\vec{G}_{k}^{\prime \prime} \times \vec{K}_{2}$ is Hamiltonian. The following remark explains the difficulty in proving that $\vec{G}_{k}^{\prime \prime}$ is bieulerian.

Remark 3.2 In fact, we know a method by which we can prove that $\vec{G}_{k}^{\prime \prime}$ is bieulerian. But proving $\vec{G}_{k}^{\prime \prime}$ is bieulerian is complicated and too long and hence we have omitted the proof. A sketch of the proof of $\vec{G}_{k}^{\prime \prime}$ is bieulerian using a "reduction technique" is described in the Appendix I of the Ph.D. thesis of the second author, see [24]. Also, using the technique described therein, we have proved that $G_{8}^{\prime \prime}$ is bieulerian, see Appendix II of [24]. This proves that $W_{8,2^{8}}$ is Hamilton cycle decomposable. Using the method described in the Appendix I mentioned above, and Lemma 3.3, of this paper, here we obtain three arc disjoint spanning bieulerian subdigraphs of $\vec{G}_{6}^{\prime}$, see Figures 8,9 and 10. Thus $W_{6,2^{6}}=W_{6,64}$ is Hamilton cycle decomposable, by Theorem 2.2. We have not given the arc disjoint bieulerian subdigraphs of $G_{k}^{\prime}$ for some smaller values of $k \geq 7$ as they have too many vertices and edges to draw.

We present three edge disjoint Hamilton cycles of $W_{6,2^{6}}$, where we assume that $(X, Y)$ is the bipartition with $X=\{(1, j) \mid 0 \leq j \leq 31\}$ and $Y=\{(2, j) \mid 0 \leq j \leq 31\}$. The three Hamilton cycles $H_{1}, H_{2}$ and $H_{3}$ of $W_{6,64}$ given below are obtained using the arc disjoint bieulerian subdigraphs (shown in Figures 8 to 10$)$ of $G_{6}^{\prime}$. 

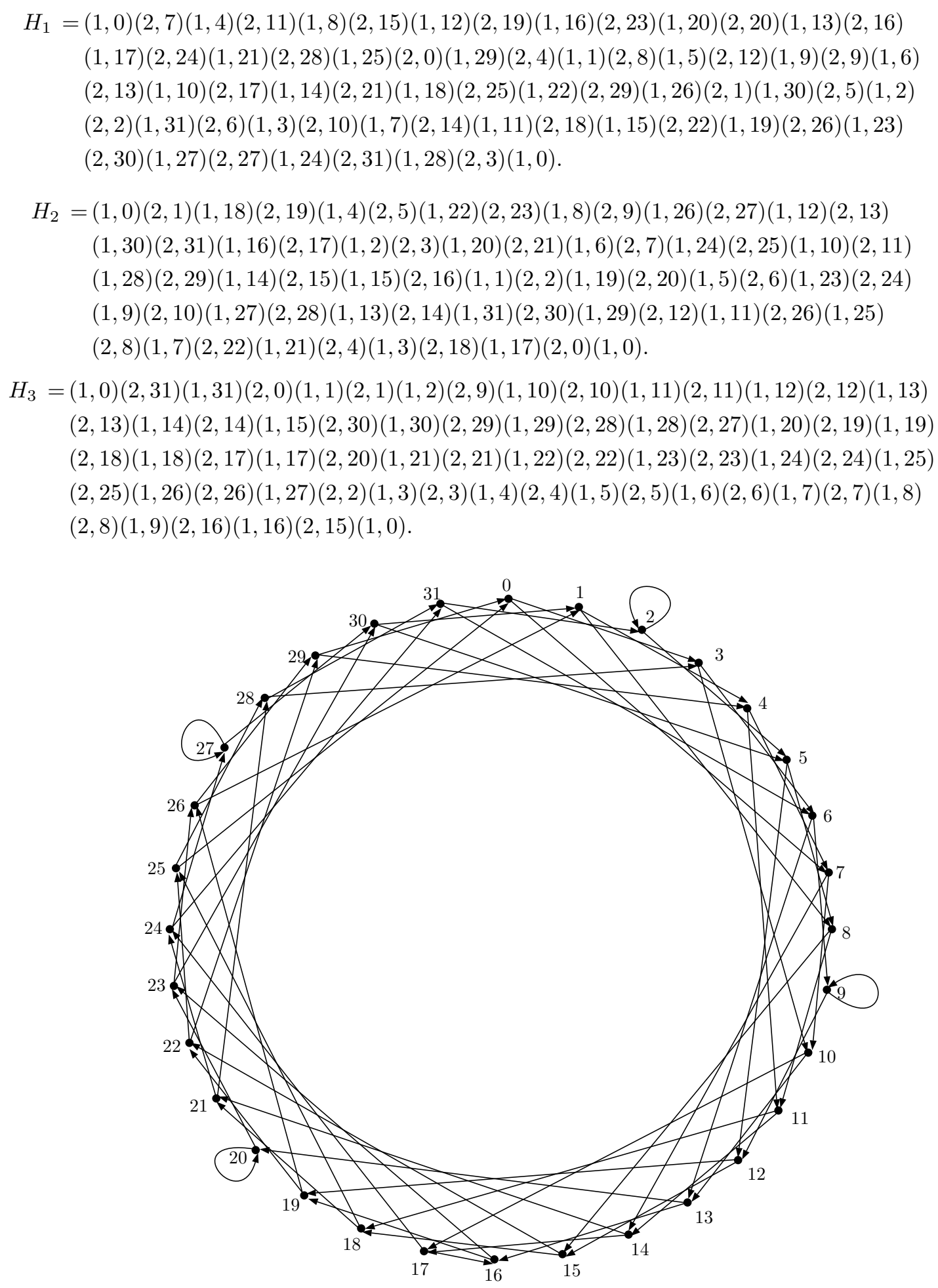

Fig. 8: A spanning 2-diregular bieulerian subdigraph $\vec{H}_{2}$ of $\vec{G}_{6}^{\prime}$. 


\section{Acknowledgements}

The second author would like to thank the Department of Science and Technology, Government of India, New Delhi and Annamalai University for awarding Junior Research Fellowship under the PURSE Programme. The second author also thank the Management and Principal, SSN College of Engineering, Chennai.

\section{References}

[1] R. Ahlswede, L. Gargano, H.S. Haroutunian and L.H. Khachatrian, Fault-Tolerant Minimum Broadcast Networks, Networks 27 (1996) 293-307.

[2] R. Balakrishnan and K. Ranganathan, A Textbook of Graph Theory, Second Edition, Springer, New York, 2012.

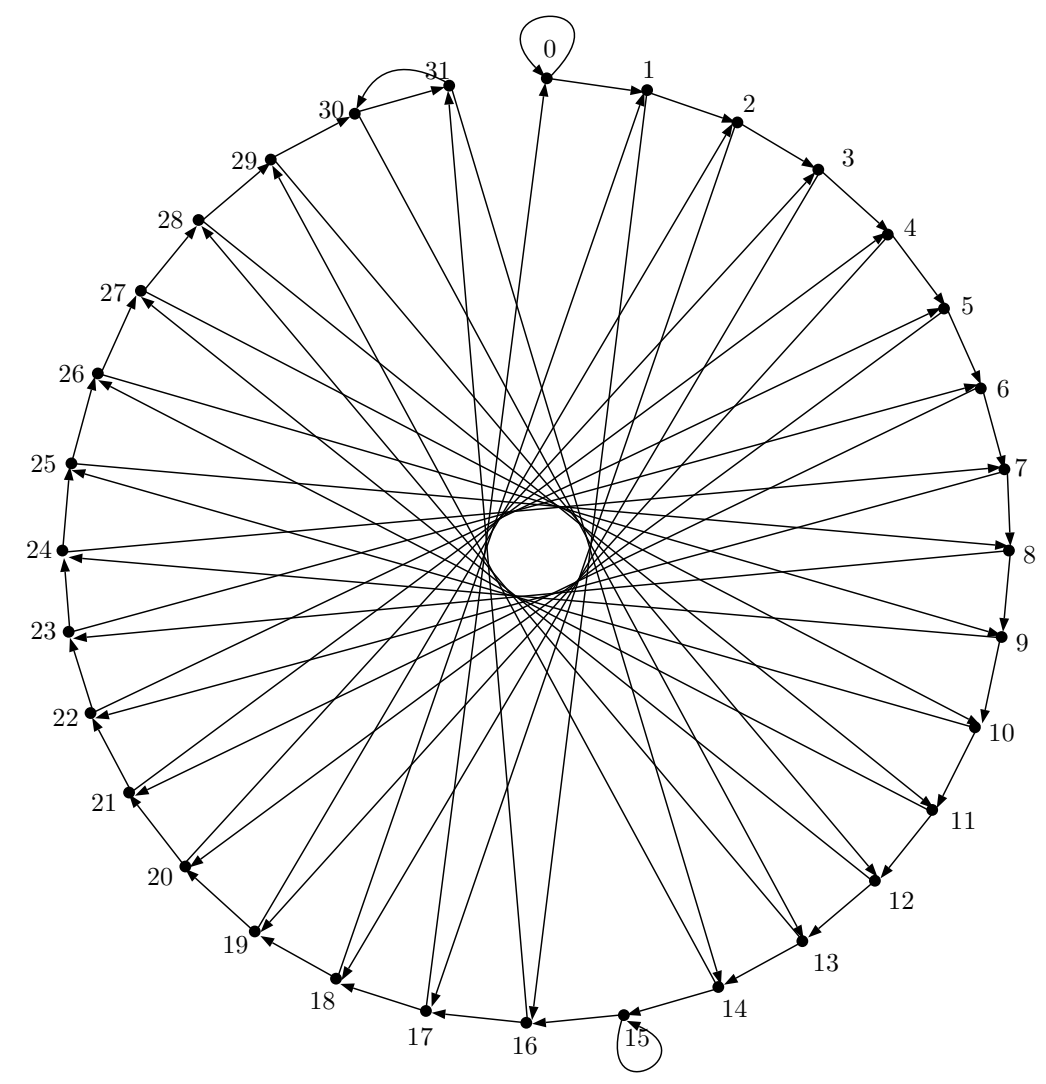

Fig. 9: A spanning 2-diregular bieulerian subdigraph $\vec{H}_{1}$ of $\vec{G}_{6}^{\prime}$. 
[3] R. Balakrishnan and P. Paulraja, Hamilton cycles in tensor product of graphs, Discrete Math. 186 (1998) 1-13.

[4] R. Balakrishnan, P. Paulraja, W. So and Vinay, The automorphism group of the Knödel graph $W_{k, 2^{k}}$ (submitted).

[5] J.-C. Bermond, H. A. Harutyunyan, A. L. Liestman and S. Perennes, A Note on the Dimensionality of Modified Knödel Graphs, Int. J. Found. Comput. Sci. 8 (1997) 109-116.

[6] J. A. Bondy and U. S. R. Murty, Graph Theory with Applications, The MacMillan Press Ltd., London, 1976.

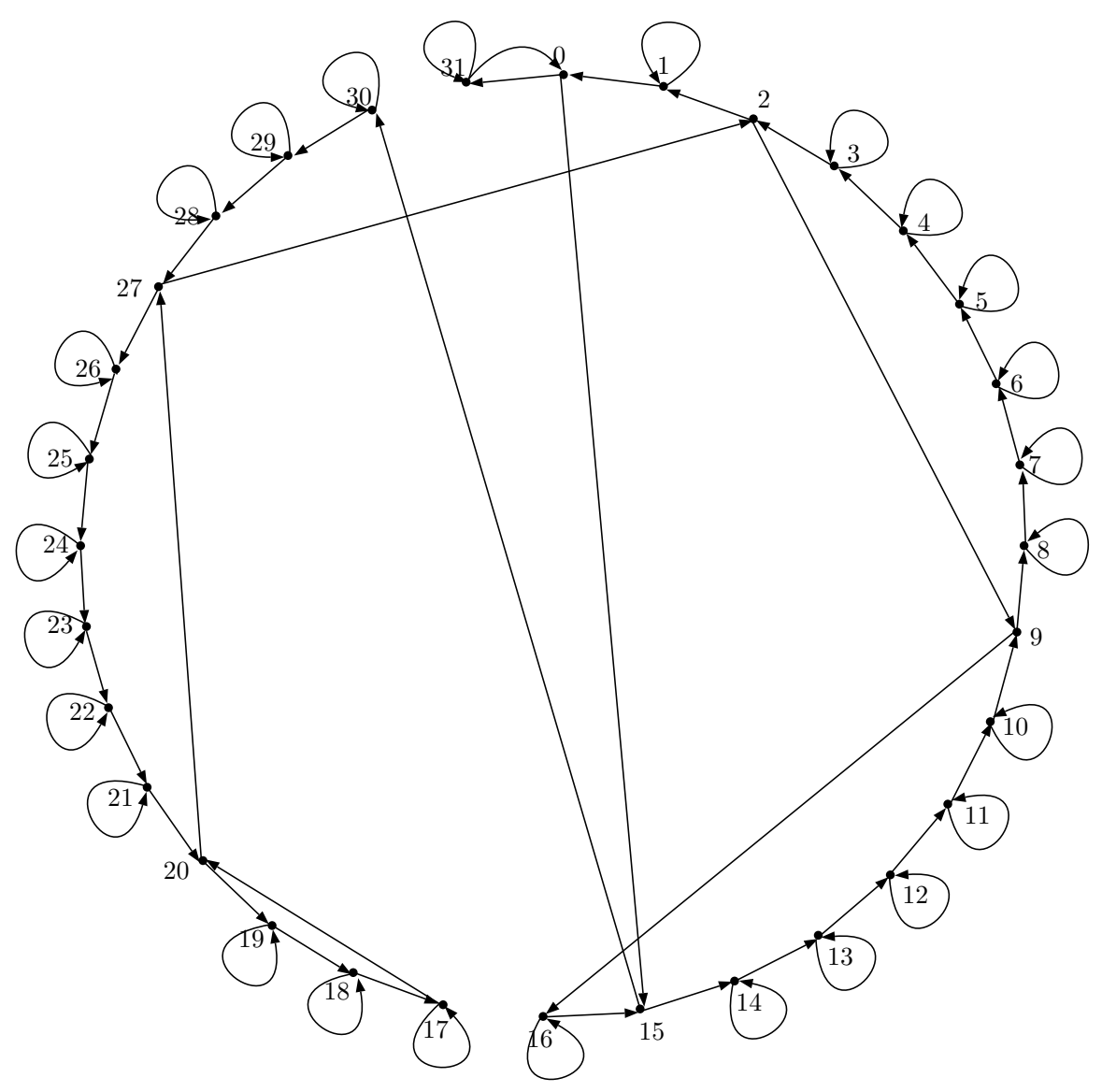

Fig. 10: A spanning 2-diregular bieulerian subdigraph $\vec{H}_{0}$ of $\vec{G}_{6}^{\prime}$. 
[7] P. Fraigniaud and J.G. Peters, Minimum linear gossip graphs and maximal linear $(\Delta, k)$-gossip graphs, Networks 38 (2001) 150-162.

[8] G. Fertin and A. Raspaud, A survey on Knödel graphs, Discrete Appl. Math. 137 (2004) 173-195.

[9] G. Fertin, A. Raspaud, H. Schröder, O. Sýkora, and I. Vrt'o, Diameter of the Knödel graph. In U. Brandes and D. Wagner, editors, Proc. 26th International Workshop on Graph-Theoretic Concepts in Computer Science (WG 2000), Vol. 1928 of LNCS, pp.149-160. Springer, Berlin, 2000.

[10] G. Fertin and A. Raspaud, Families of graphs having broadcasting and gossiping properties, in: Proceedings of the 24th International Workshop on Graph-Theoretic Concepts in Computer Science (WG' 98), Vol. 1517, Smolenice, LNCS, 1998, pp. 63-77.

[11] G. Gauyacq, Routages uniformes dans les graphes sommet-transitifs, 1995.

[12] H. Grigoryan and H. A. Harutyunyan, Tight Bound on the Diameter of the Knödel Graph, IWOCA 2013, 206-215.

[13] H. Grigoryan and H. A. Harutyunyan, The shortest path problem in the Knödel graph, J. Discrete Algorithms 31 (2015) 40-47.

[14] H.A. Harutyunyan, Minimum multiple message Broadcast graphs, Networks 47 (2006) 218-224.

[15] H. A. Harutyunyan, Multiple message broadcasting in modified Knödel graph, SIROCCO 2000, 157-165.

[16] H. A. Harutyunyan and C. D. Morosan, The spectra of Knödel graphs, Informatica 30 (2006) 295299.

[17] H. A. Harutyunyan and C. D. Morosan, On the minimum path problem in Knödel graphs, Networks 50 (2007) 86-91.

[18] H.A. Harutyunyan and A.L. Liestman, Upper bounds on the broadcast function using minimum dominating sets, Discrete Math. 312 (2012) 2992-2996.

[19] S.M. Hedetniemi, S.T. Hedetniemi and A.L. Liestman, A survey of gossiping and broadcasting in communication networks. 18 (1988) 319-349.

[20] J. Hromkovič, R. Klasing, B. Monien and R. Peine, Dissemination of information in interconnection networks (broadcasting and gossiping). In, D.-Z. Du and D.F. Hsu, Editors, Combinatorial Network Theory, pp. 125-212, Kluwer Academic Publishers, 1996.

[21] L.H. Khachatrian and H.S. Haroutunian, Construction of new classes of minimal broadcast networks, Proceedings of the Third International Colloquium on Coding Theory, 1990, pp. 69-77.

[22] W. Knödel, New gossips and telephones, Discrete Math. 13 (1975) 95.

[23] R. Labahn, Some minimum gossip graphs, Networks 23 (1993) 333-341.

[24] S. Sampath Kumar, Decompositions of Graphs into Trails and Cycles, Ph.D. thesis, Annamalai University, India, 2013. 